\title{
A geometrical approach to front propagation problems in bounded domains with Neumann-type boundary conditions
}

\author{
GUY BARLES ${ }^{\dagger}$ \\ Laboratoire de Mathématiques et Physique Théorique, Faculté des Sciences et Techniques, \\ Université de Tours, Parc de Grandmont, 37200 Tours, France \\ AND \\ FRANCESCA DA LIO \\ Dipartimento di Matematica, Università di Torino \\ Via Carlo Alberto 10, 10123 Torino, Italy
}

[Received 30 November 2001 and in revised form 17 March 2003]

\begin{abstract}
We are interested in the asymptotic behavior of the solutions of scaled reaction-diffusion equations in bounded domains, associated with Neumann type boundary conditions, and more precisely in cases when such behavior is described in terms of moving interfaces. A typical example is the case of the Allen-Cahn equation associated with an oblique derivative boundary condition, where the generation of a front moving by mean curvature with an angle boundary condition is shown. In order to establish such results rigourously, we modify and adapt the "geometrical approach" introduced by P. E. Souganidis and the first author for solving problems in $\mathbb{R}^{N}$ : we provide a new definition of weak solution for the global-in-time motion of fronts with curvature-dependent velocities and with angle boundary conditions, which turns out to be equivalent to the level-set approach when there is no fattening phenomenon. We use this definition to obtain the asymptotic behavior of the solutions of a large class of reaction-diffusion equations, including the case of quasilinear ones and $(x, t)$ dependent reaction terms, but also with any, possibly nonlinear, Neumann boundary conditions.
\end{abstract}

Keywords: Front propagation; reaction-diffusion equations; asymptotic behavior; geometrical approach; level-set approach; Neumann boundary condition; angle boundary condition; viscosity solutions.

2000 Mathematics Subject Classification: 35B05, 35B40, 35K57, 53C44, 35K60, 35B25, 49L25.

\section{Introduction}

Front propagation phenomena can be observed in a lot of physical, chemical or biological situations: flame propagation in combustion, phase transitions, evolution of populations or spreading of diseases etc. From a mathematical point of view, they appear naturally in the study of asymptotic limits of evolving systems, like reaction-diffusion equations or particle systems.

In the past fifteen years, a lot of work has been devoted to rigourously establish the connections between reaction-diffusion equations or particle systems with the wavefronts they generate. In order to do it, two kinds of difficulties had to be solved: the first key problem was to obtain a suitable

\footnotetext{
†Email: barles@univ-tours.fr

Email: dalio@dm.unito.it
} 
"weak" definition for the evolutions of hypersurfaces with prescribed normal velocities, and in particular with curvature-dependent velocities. Indeed, for the applications, the front propagations have to be defined for all time but it is well known that, in general, smooth evolutions cannot exist globally in time. The aim was both to define these motions past the development of singularities but also to have a sufficiently flexible definition in order to be able to actually study the asymptotic limits of reaction-diffusion equations or particle systems, which is the second difficulty.

Most of this work was done in the case of problems set in the whole space $\mathbb{R}^{N}$ or in related situations where no constraints were imposed on the motions. The aim of this paper is to consider the case of reaction-diffusion equations set in bounded domains with Neumann type boundary conditions which are associated to motions of fronts with angle boundary conditions on the boundary. In order to do it, we are going to slightly modify and extend the "geometrical approach" of P. E. Souganidis and the first author [6] to take into account this kind of problems.

In order to be more specific, we consider, as a model case, the example of the Allen-Cahn equation associated to an oblique derivative boundary condition. The aim is to study the asymptotics of the solutions of

$$
u_{\varepsilon, t}-\Delta u_{\varepsilon}+\varepsilon^{-2} f\left(u_{\varepsilon}\right)=0 \quad \text { in } O \times(0, \infty),
$$

where $O$ is a smooth bounded domain in $\mathbb{R}^{N}, u_{\varepsilon}: \bar{O} \times[0, \infty) \rightarrow \mathbb{R}$ is the solution and the nonlinearity $f$ is of the form $f=W^{\prime}, W$ being a double-well potential. The so-called Allen-Cahn equation corresponds to the choice

$$
f(u)=2 u\left(u^{2}-1\right) \quad \text { for } u \in \mathbb{R} .
$$

We consider (1) together with an oblique derivative boundary condition

$$
\frac{\partial u_{\varepsilon}}{\partial \gamma}=0 \quad \text { on } \partial O \times(0, \infty)
$$

where $\gamma: \partial O \times[0, \infty) \rightarrow \mathbb{R}^{N}$ is a Lipschitz continuous vector field such that $\gamma(x, t) \cdot n(x)>0$ on $\partial O \times[0, \infty), n(x)$ being the unit exterior normal vector to $\partial O$ at $x$. Finally we impose an initial data

$$
u_{\varepsilon}(x, 0)=g(x) \quad \text { on } \bar{O} \times\{0\},
$$

where $g \in C(\bar{O})$.

We recall that the Allen-Cahn equation was introduced in [1] to model the motion of the sharp interface - the antiphase boundary-between regions of different phases of a material. In $\mathbb{R}^{N}$, the formal analysis of Keller, Rubinstein and Sternberg [18] shows that the interface, i.e. the thin region separating the subsets of $\mathbb{R}^{N}$ where $u_{\varepsilon}$ is converging to the stable equilibria of the equation, is moving by mean curvature when $f$ is given by (2). A first rigourous, but partial, proof of this result was proposed by Chen [7] (both in $\mathbb{R}^{N}$ and in bounded open subsets of $\mathbb{R}^{N}$ with Neumann boundary conditions) in the case when the motion by mean curvature is classical, i.e. the fronts are smooth hypersurfaces evolving smoothly. This means in fact a small time result since it is well known that, for the motion by mean curvature, singularities develop in finite time.

In order to rigourously prove and even formulate the result for all times, a suitable notion of generalized motion by mean curvature is needed in order to define it past the development of singularities. This question was solved in a rather general way by the "level-set approach", first introduced by Osher and Sethian [19] for numerical computations and then developed from a theoretical point of view by Evans and Spruck [11] for the motion by mean curvature and by Chen, 
Giga and Goto [8] for more general, curvature-dependent motions. Then a different but related approach using the properties of the (signed) distance to the front was introduced by Soner [20] and further developed in Barles, Soner and Souganidis [5]; as we will see below, the distance function to the moving front plays a key role in the study of the asymptotics of reaction-diffusion equations. For a general review of these theories, their relationships as well as other related facts we refer to Souganidis [21,22].

Using the level-set approach and the properties of the distance function to the moving front, the asymptotics of the Allen-Cahn equation was first proved rigorously and for all times in Evans, Soner and Souganidis [10] and then by different methods in [5]. But the purely analytical methods of those papers were not very flexible and therefore not easily extendable to more complicated reactiondiffusion equations, for example equations with oscillating coefficients, and even less to general nonlocal, fully nonlinear equations such as the ones appearing in the study of particle systems. In particular they were not adapted to take into account front propagations in which the velocity depends on the normal direction.

To solve this problem, a different approach, more geometrical, was introduced by Souganidis and the first author in [6]. Based on a new definition of the generalized propagation of fronts in $\mathbb{R}^{N}$ which turns out to be equivalent to the level-set approach when there is no fattening phenomenon, it leads to a simple and general method for establishing the asymptotic limit of a large class of reaction-diffusion equations and particle systems. Roughly speaking, this method reduces the study of such asymptotics to the cases when the evolving front is smooth and evolves smoothly, which means, at the practical level, for small time.

Before describing the approach of [6] and our modification for problems with Neumann type boundary conditions, we recall that the level-set approach for such problems was first considered for the classical homogeneous Neumann boundary condition in Giga and Sato [15]. Extensions to nonlinear Neumann boundary conditions were obtained in Barles [3] and in Ishii and Sato [16] under different conditions on the regularity of the domain and on the boundary condition. A nonlinear Neumann boundary condition is of the form

$$
G(x, t, D u)=0 \quad \text { on } \partial O \times(0, \infty),
$$

where $G: \partial O \times(0, \infty) \times \mathbb{R}^{N} \rightarrow \mathbb{R}$ is a continuous function satisfying: for any $T>0$, there exists a constant $v(T)>0$ such that, for all $\lambda>0, x \in \partial O, t \in[0, T], p \in \mathbb{R}^{N}$, one has

$$
G(x, t, p+\lambda n(x))-G(x, t, p) \geqslant v(T) \lambda .
$$

In addition to this property which is characteristic of the Neumann boundary condition, one has to assume that $G$ is homogeneous of degree 1 in $p$, which is a geometrical condition, i.e. a condition for the level-set approach to work.

The classical homogeneous Neumann or oblique derivative boundary conditions are examples of boundary conditions satisfying these conditions but there are also nonlinear boundary conditions like the following capillarity type boundary condition:

$$
\frac{\partial u}{\partial n}=\theta(x, t)|D u| \quad \text { on } \partial O \times(0, \infty),
$$

where $\theta: \partial O \times[0, \infty) \rightarrow \mathbb{R}$ is, say, a locally Lipschitz continuous function such that $|\theta(x, t)|<1$ on $\partial O \times[0, \infty)$. 
Of course, for degenerate and singular parabolic equations in the level-set approach, these boundary conditions have to be considered in the viscosity sense; we refer to the user's guide of viscosity solutions of Crandall, Ishii and Lions [9] or to the book of Fleming and Soner [13] for a presentation and discussion of boundary conditions in the viscosity solutions sense (see also Barles [3]).

We recall that a global-in-time result on the asymptotics of the Allen-Cahn equation in bounded domains with Neumann boundary conditions was obtained by Katsoulakis, Kossioris and Reitich [17] under the assumption that the domain is convex.

Now we come back to the approach of [6]; it consists in first considering the evolution of open subsets of $\mathbb{R}^{N}$ instead of hypersurfaces. From the point of view of applications, this idea is very natural since the moving front is just an evolving interface separating regions where the system is close to one of its equilibria and therefore it is even more natural to study the evolution of these regions. This approach relies on the "monotonicity property" of the front propagations, also called "avoidance principle" for the mean curvature motion. Roughly speaking, this monotonicity property is expressed in the following way: if $\left(\Omega_{s}^{1}\right)_{s \in(a, b)},\left(\Omega_{s}^{2}\right)_{s \in(a, b)}$ are two families of open subsets evolving with the same normal velocity and if $\Omega_{t}^{1} \subset \Omega_{t}^{2}$ for some $t \in(a, b)$, then

$$
\Omega_{s}^{1} \subset \Omega_{s}^{2} \quad \text { for any } s \in[t, b) .
$$

This property can be seen for example as a consequence of the maximum principle for the levelset pde, and the main remark in [6] is that it can be used as a definition for weak motions: roughly speaking, one may say that a family $\left(\Omega_{s}^{2}\right)_{s \in(a, b)}$ has a generalized motion with normal velocity greater than $V_{n}$ if it satisfies the above monotonicity property when tested on a sufficiently large class of families $\left(\Omega_{s}^{1}\right)_{s \in(a, b)}$ evolving with normal velocity less than $V_{n}$. A notion of generalized motion with normal velocity less than $V_{n}$ can be defined analogously, but because it is easier to deal with families of "small open test subsets", a passage to the complement turns out to be more convenient. The key points used in [6] are then that (i) it is enough to test against families of smooth open subsets evolving smoothly, (ii) this has to be done only on a small time interval and (iii) as described above, one can use families whose normal velocities are smaller or greater than the normal velocity considered, if we do it in a suitable way.

At this level of generality, these basic ideas apply more or less readily in our framework since the level-set approach enjoys the same kind of monotonicity properties in the Neumann case as the $\mathbb{R}^{N}$ case. But we face several difficulties in concrete applications. First, in [6], the "open test subsets" were taken of the form $\{x: \phi(x, s)>0\}$ where the function $\phi$ was either a strict subsolution or supersolution of the level-set equation at least in a neighborhood of $\{x: \phi(x, s)=0\}$. Such functions $\phi$ were built from $\phi(\cdot, 0)$ by using an Euler type scheme or a small time existence result for smooth solutions of the level-set pde. In our case, because of the Neumann type boundary condition, the use of the Euler scheme was impossible and not many existence results for smooth solutions were available, at least to the best of our knowledge.

To overcome this difficulty, we modify the definition given in [6], and it is worth pointing out that these modifications are a key step to solving the main problems we face with the additional boundary condition. As in [6] we localize the monotonicity property by considering balls $B(x, r)$ for $x \in \bar{\Omega}$ but we drop the condition that the moving front $\{y: \phi(y, s)=0\}$ has to be included in $B(x, r)$, a condition which was not very natural. To do that, we have to impose a condition on the boundary of the ball and the monotonicity is expressed in the following way: if, for some $t \in(a, b)$,

$$
\Omega_{t}^{1} \cap B(x, r) \subset \Omega_{t}^{2} \cap B(x, r),
$$


AND if, for all $t \leqslant s<b$, we have

$$
\Omega_{s}^{1} \cap \partial B(x, r) \subset \Omega_{s}^{2} \cap \partial B(x, r),
$$

then, for any $t \leqslant s<b$,

$$
\Omega_{s}^{1} \cap B(x, r) \subset \Omega_{s}^{2} \cap B(x, r) .
$$

The new condition (7) can be viewed as a Dirichlet type boundary condition.

But the main difference in comparison with the definition of [6] is the way in which we define the family of "open test subsets": as in [6], they are of the form $\{x: \phi(x, s)>0\}$ but here we reduce to the case where we already know that $\phi$ is either a strict subsolution or strict supersolution of the level-set equation satisfying also the Neumann boundary condition in a strict sense. This avoids delicate constructions of such strict sub- or supersolutions and a consequence is that we do not need any more to invoke "small time" arguments which were in fact related to these constructions. The introduction of the above mentioned "Dirichlet type boundary condition" on the front allows us to make this reduction.

With this new definition, we are able to provide new and rather general results on the asymptotics of reaction-diffusion equations: we extend all the applications treated in [6] and [5], namely semilinear and quasilinear type Allen-Cahn equations with a possible $(x, t)$-dependence in the nonlinearity $f$, to the case when these equations are set in a bounded domain with Neumann type boundary conditions. In particular, we show here that, in the case of (1)-(3)-(4), the interface still moves by mean curvature but with an angle boundary condition on $\partial O$.

Even the proofs of [6] extend almost readily, with however two important modifications: first, in order to take into account the Dirichlet type boundary condition on $\partial B(x, r)$, we have to define in a different way the family of open subsets $\left(\Omega_{s}\right)_{s \in(a, b)}$ about which we aim to prove that they move with a certain normal velocity. In [6], these open subsets were, roughly speaking, the interiors of the sets where $u_{\varepsilon}$ converges to the stable equilibria of the equation; here we have to define them as the interiors of the sets where this convergence holds with an $o\left(\varepsilon^{\tau}\right)$ rate of convergence where $\tau$ depends on the problem and is typically equal to 1 for curvature-dependent motions. The second point concerns the proof of the so-called "propagation of the interface": in [6], almost all the asymptotic results were obtained by building sub- and supersolutions of reaction-diffusion equations by using directly the test function $\phi$ or, in more complicated cases, the (signed) distance to the moving front $\{x: \phi(x, s)=0\}$. Here, because of the Neumann boundary condition, we have to use the distance function systematically. However, it is worth pointing out that this distance function is an $\mathbb{R}^{N}$-distance function associated to propagation in $\mathbb{R}^{N}$ and not a distance relative to $\bar{O}$; this will of course simplify matters.

Finally we emphasize that there is not much difference between treating nonlinear Neumann type boundary conditions, for example capillarity type boundary conditions like (6), and the case of (linear) homogeneous Neumann or oblique derivative. This is another advantage of our approach.

The only examples of [6] we are not able to extend to our framework are the ones related to reaction-diffusion equations with oscillating coefficients. The problem we face has nothing to do with our approach but is deeper: in this case we are lacking the formal asymptotic behavior of the solution that we use in a fundamental way for building sub- and supersolutions, and we do not know how to build them. From our point of view, this is a very challenging open question which does not concern only front propagation problems but also homogenization problems. Such kind of difficulties arise, for example, in the homogenization of first-order Hamilton-Jacobi equations with 
Neumann boundary conditions, and also in some second-order elliptic or parabolic equations with a suitable dependence on $\varepsilon$.

The paper is organized as follows: Section 1 is devoted to the presentation of the new definition for motions with angle boundary conditions and its connections with the level-set approach. Section 2 is devoted to the application of the new definition to the study of the asymptotics of reactiondiffusion equations: we first present a general abstract method and then we apply it to the model case of the Allen-Cahn equation with a nonlinear Neumann derivative boundary condition; finally we present the extensions concerning the asymptotics of quasilinear reaction-diffusion equations with $x, t$ and $\varepsilon$-dependent $f$ 's.

\section{A new geometric definition and its connections with the level-set approach}

The aim of this section is to develop a new approach to the weak geometric motion of hypersurfaces in bounded domains with prescribed normal velocity and angle boundary condition and to show its connections with the level-set approach.

We first briefly recall the basic ideas of the level-set approach. Let $O \subset \mathbb{R}^{N}$ be a smooth bounded open set, let $F$ be a real-valued, locally bounded function on $\bar{O} \times(0, \infty) \times \mathbb{R}^{N} \times \mathcal{S}(N)$, which is continuous on $\bar{O} \times(0, \infty) \times\left(\mathbb{R}^{N} \backslash\{0\}\right) \times \mathcal{S}(N), \mathcal{S}(N)$ being the set of real symmetric $N \times N$ matrices, and let $G$ be a real-valued, continuous function on $\partial O \times(0, \infty) \times \mathbb{R}^{N}$. We consider the following initial-value problem with a nonlinear Neumann boundary condition:

$$
\begin{cases}\text { (i) } u_{t}+F\left(x, t, D u, D^{2} u\right)=0 & \text { in } O \times(0, T), \\ \text { (ii) } G(x, t, D u)=0 & \text { in } \partial \times(0, T), \\ \text { (iii) } u(x, 0)=u_{0}(x) & \text { in } \bar{O},\end{cases}
$$

where, in (8)(ii), we have typically in mind the two cases $(3)$ and $(6)$.

For the level-set approach to work, we first need an existence and comparison result for (8). For simplicity, we do not present here the technical assumptions which are used to prove such results and refer instead to [3, 15, 16]. Among all those assumptions, we want to point out anyway the following basic assumptions on $F$ and $G$ :

(A1) The function $F$ is a real-valued, locally bounded function on $\bar{O} \times(0, \infty) \times \mathbb{R}^{N} \times \mathcal{S}(N)$, continuous on $\bar{O} \times(0, \infty) \times \mathbb{R}^{N} \backslash\{0\} \times \mathcal{S}(N)$ and satisfying the ellipticity condition

$$
F(x, t, p, X) \leqslant F(x, t, p, Y) \quad \text { whenever } X \geqslant Y,
$$

for any $x \in \bar{O}, t \in(0, \infty), p \in \mathbb{R}^{N} \backslash\{0\}$ and $X, Y \in \mathcal{S}(N)$, where " $\geqslant$ " stands for the usual partial ordering on symmetric matrices.

(A2) The function $G$ is uniformly continuous on $\partial O \times(0, \infty) \times \mathbb{R}^{N}$ and for any $T>0$ there exists a constant $\nu(T)>0$ such that, for all $\lambda>0, x \in \partial O, t \in[0, T]$ and $p \in \mathbb{R}^{N}$,

$$
G(x, t, p+\lambda n(x))-G(x, t, p) \geqslant v(T) \lambda,
$$

where $n(x)$ is the unit exterior normal vector to $\partial O$ at $x \in \partial O$.

Assumption (A1) is a key hypothesis to use viscosity solutions, (A2) characterizes suitable nonlinear Neumann boundary conditions. 
On the other hand, we need specific assumptions related to the geometric aspect of the problem; they are the following:

(A3) For any $\lambda>0, v \in \mathbb{R}$ and $x \in \bar{O}, t \in(0, \infty), p \in \mathbb{R}^{N} \backslash\{0\}, X \in \mathcal{S}(N)$,

$$
F(x, t, \lambda p, \lambda X+v p \otimes p)=\lambda F(x, t, p, X)
$$

where $p \otimes p$ denotes the symmetric matrix defined by $(p \otimes p)_{i j}=p_{i} p_{j}$ for all $1 \leqslant i, j \leqslant N$.

(A4) For all $\lambda>0, x \in \partial O, t \in(0, \infty)$ and $p \in \mathbb{R}^{N}$,

$$
G(x, t, \lambda p)=\lambda G(x, t, p) .
$$

We just notice here that the main consequence of (A3) and (A4) is that if $u$ is a solution of (8) then $\chi(u)$ is also a solution of $(8)$ for any map $\chi: \mathbb{R} \rightarrow \mathbb{R}$ such that $\chi^{\prime}>0$ in $\mathbb{R}$.

The whole set of assumptions which implies, on the one hand, existence and uniqueness of a continuous solution of 8 for any $u_{0} \in C(\bar{O})$ and a comparison result between sub- and supersolutions of 88 , together with (A3) and (A4), will be referred to below as the "assumptions of the level-set approach".

The level-set approach for problems associated with Neumann type boundary conditions (see e.g. [3, 15, 16]) can be described in a similar way to the $\mathbb{R}^{N}$ case (see e.g. [8, 11]). Let $\mathcal{E}$ be the collection of triplets $\left(\Gamma, D^{+}, D^{-}\right)$of mutually disjoint subsets of $O$ such that $\Gamma$ is closed, $D^{ \pm}$are open and $\bar{O}=\Gamma \cup D^{+} \cup D^{-}$. For any $\left(\Gamma_{0}, D_{0}^{+}, D_{0}^{-}\right) \in \mathcal{E}$, first choose $u_{0} \in C(\bar{O})$ (the space of continuous functions defined on $\bar{O}$ ) so that

$$
D_{0}^{+}=\left\{x \in \bar{O}: u_{0}(x)>0\right\}, \quad D_{0}^{-}=\left\{x \in \bar{O}: u_{0}(x)<0\right\}, \quad \Gamma_{0}=\left\{x \in \bar{O}: u_{0}(x)=0\right\},
$$

By results of [3, 15, 16], for every $u_{0} \in C(\bar{O})$, there exists a unique viscosity solution $u$ of $(8)$ in $C(\bar{O} \times[0, \infty))$. If, for all $t>0$, we define $\left(\Gamma_{t}, D_{t}^{+}, D_{t}^{-}\right) \in \mathcal{E}$ by

$$
\Gamma_{t}=\{x \in \bar{O}: u(x, t)=0\}, \quad D_{t}^{+}=\{x \in \bar{O}: u(x, t)>0\}, \quad D_{t}^{-}=\{x \in \bar{O}: u(x, t)<0\},
$$

then, because of (A3), (A4) and since a comparison result holds for (8), the collection $\left\{\left(\Gamma_{t}, D_{t}^{+}, D_{t}^{-}\right)\right\}_{t \geqslant 0}$ is uniquely determined, independently of the choice of $u_{0}$, by the initial triplet $\left(\Gamma_{0}, D_{0}^{+}, D_{0}^{-}\right)$.

The properties of the generalized level evolution have been the object of extensive study, at least in $\mathbb{R}^{N}$. One of the most intriguing issues-rather important in the study of the asymptotics of reaction-diffusion equations-is whether the so-called fattening phenomenon occurs, i.e. whether the set $\bigcup_{t>0} \Gamma_{t} \times\{t\}$ has an interior.

Following the $\mathbb{R}^{N}$-case, we say that the no-interior condition holds for the set $\{u=0\}$ if

$$
\{(x, t): u(x, t)=0\}=\partial\{(x, t): u(x, t)>0\}=\partial\{(x, t): u(x, t)<0\} .
$$

The question of whether 13 holds was discussed in $\mathbb{R}^{N}$ in [5] (see also references therein): conditions are given on $\Gamma_{0}$ and the equation ensuring that (13) is satisfied as well as examples where it fails. Two examples of fattening for the Neumann problem have been provided by G. Barles in [3] and Y. Giga in [14].

The importance of the no-interior condition and its connection with more geometrical approaches than the level-set approach are explained in the following result, proved in $\mathbb{R}^{N}$ in [5], 
and which extends easily to the case of Neumann boundary conditions. In this result, if $A$ is a subset of some $\mathbb{R}^{k}$, then $\mathbb{1}_{A}$ denotes the indicator function of $A$, i.e., $\mathbb{1}_{A}(x)=1$ if $x \in A$ and $\mathbb{1}_{A}(x)=0$ if $x \in A^{c}$.

THEOREM 1.1 Under the assumptions of the level-set approach, the functions $\mathbb{1}_{D_{t}^{+} \cup \Gamma_{t}}-\mathbb{1}_{D_{t}^{-}}$ and $\mathbb{1}_{D_{t}^{+}}-\mathbb{1}_{D_{t}^{-} \cup \Gamma_{t}}$ are respectively the maximal subsolution (and solution) and the minimal supersolution (and solution) of 8 associated respectively with the initial data $u_{0}=\mathbb{1}_{D_{0}^{+} \cup \Gamma_{0}}-\mathbb{1}_{D_{0}^{-}}$ and $u_{0}=\mathbb{1}_{D_{0}^{+}}-\mathbb{1}_{D_{0}^{-} \cup \Gamma_{0}}$. Moreover, if $\Gamma_{0}$ has an empty interior, then $\mathbb{1}_{D_{t}^{+}}-\mathbb{1}_{D_{t}^{-}}$is the unique discontinuous solution of $\sqrt{8}$ associated with the initial data $u_{0}=\mathbb{1}_{D_{0}^{+}}-\mathbb{1}_{D_{0}^{-}}$if and only if the property 13 holds.

In fact the main consequence of this result is that if $(13)$ holds, then the problem is well-posed in the geometrical sense since the evolution of the indicator function (or equivalently of the underlying sets) is uniquely determined.

Now we turn to the geometrical definition in the case of Neumann boundary conditions. To simplify the presentation, we have to introduce some notations. If $A$ is a subset of some $\mathbb{R}^{k}$, we denote by $\operatorname{Int}(A)$ the interior of $A$, and if $x \in A$ and $r>0$, we set $B_{A}(x, r):=B(x, r) \cap A$ (the open ball in the topology of $A), \bar{B}_{A}(x, r):=\bar{B}(x, r) \cap A$ (the closed ball in the topology of $A$ ) and $\partial B_{A}(x, r):=\partial B(x, r) \cap A$.

In what follows we denote by $\left(\Omega_{t}\right)_{t \in(0, T)}$ a family of open subsets of $\bar{O}$ and we set $\Gamma_{t}=\partial \Omega_{t}$. The signed-distance function $d(x, t)$ from $x$ to $\Gamma_{t}$ is defined by

$$
d(x, t)= \begin{cases}d\left(x, \Gamma_{t}\right) & \text { if } x \in \Omega_{t}, \\ -d\left(x, \Gamma_{t}\right) & \text { otherwise }\end{cases}
$$

where $d\left(x, \Gamma_{t}\right)$ denotes the usual nonnegative distance from $x \in \mathbb{R}^{N}$ to $\Gamma_{t}$. If $\Gamma_{t}$ is a smooth hypersurface, then $d$ is a smooth function in a neighborhood of $\Gamma_{t}$, and for $x \in \Gamma_{t}, n(x, t)=$ $-D d(x, t)$ is the unit normal to $\Gamma_{t}$ pointing away from $\Omega_{t}$.

Finally, we recall that for a locally bounded function $f: A \rightarrow \mathbb{R}$, where $A$ is a subset of some $\mathbb{R}^{k}$, the upper and lower semicontinuous envelopes $f^{*}$ and $f_{*}$ of $f$ are given by

$$
f^{*}(y)=\limsup _{z \rightarrow y} f(z) \text { and } \quad f_{*}(y)=\liminf _{z \rightarrow y} f(z) .
$$

Now we give the definition of generalized super- and subflow in bounded domains with a prescribed normal velocity and angle boundary condition.

Definition 1.1 A family $\left(\Omega_{t}\right)_{t \in(0, T)}$ (resp. $\left.\left(\mathcal{F}_{t}\right)_{t \in(0, T)}\right)$ of open (resp. closed) subsets of $\bar{O}$ is called a generalized superflow (resp. subflow) with normal velocity $-F\left(x, t, D d, D^{2} d\right.$ ) and angle condition $G(x, t, D d)$ if, for any $x_{0} \in \bar{O}, t \in(0, T), r>0, h>0$ and for any smooth function $\phi: \bar{O} \times[0, T] \rightarrow \mathbb{R}$ such that

(i) $\partial \phi / \partial t+F^{*}\left(y, s, D \phi, D^{2} \phi\right)<0\left(\right.$ resp. $\left.\partial \phi / \partial t+F_{*}\left(y, s, D \phi, D^{2} \phi\right)>0\right)$ in $\bar{B} \bar{O}\left(x_{0}, r\right) \times$ $[t, t+h]$

(ii) $G(y, s, D \phi)<0$ (resp. $G(y, s, D \phi)>0)$ in $\partial O \cap \bar{B}\left(x_{0}, r\right) \times[t, t+h]$,

(iii) for any $s \in[t, t+h],\left\{y \in B \bar{O}\left(x_{0}, r\right): \phi(y, s)=0\right\} \neq \emptyset$ and

$$
|D \phi(y, s)| \neq 0 \quad \text { on }\left\{(y, s) \in \bar{B} \bar{O}\left(x_{0}, r\right) \times[t, t+h]: \phi(y, s)=0\right\}
$$


(iv) $\left\{y \in \bar{B} \bar{O}\left(x_{0}, r\right): \phi(y, t) \geqslant 0\right\} \subset \Omega_{t}\left(\right.$ resp. $\left.\left\{y \in \bar{B} \bar{O}\left(x_{0}, r\right): \phi(y, t) \leqslant 0\right\} \subset \mathcal{F}_{t}^{c}\right)$,

(v) for all $s \in[t, t+h]$,

$$
\begin{aligned}
\{y & \left.\in \partial B \bar{O}\left(x_{0}, r\right): \phi(y, s) \geqslant 0\right\} \subset \Omega_{s} \\
(\text { resp. }\{y & \left.\left.\in \partial B \bar{O}\left(x_{0}, r\right): \phi(y, s) \leqslant 0\right\} \subset \mathcal{F}_{s}^{c}\right),
\end{aligned}
$$

we have

$$
\begin{aligned}
&\{y\left.\in B_{\bar{O}}\left(x_{0}, r\right): \phi(y, t+h)>0\right\} \subset \Omega_{t+h} \\
&\text { (resp. } \left.\left\{y \in B_{\bar{O}}\left(x_{0}, r\right): \phi(y, t+h)<0\right\} \subset \mathcal{F}_{t+h}^{c}\right) .
\end{aligned}
$$

A family $\left(\Omega_{t}\right)_{t \in(0, T)}$ of open subsets of $\bar{O}$ is called a generalized flow with normal velocity $-F\left(x, t, D d, D^{2} d\right)$ and angle boundary condition $G(x, t, D d)$ if $\left(\Omega_{t}\right)_{t \in(0, T)}$ is a superflow and $\left(\bar{\Omega}_{t}\right)_{t \in(0, T)}$ is a subflow.

As mentioned in the introduction, the main difference compared to the definition of generalized sub- and superflow introduced in [6] is that we use functions $\phi$ already defined in $\bar{O} \times[0, T]$ and which are either sub- or supersolutions of the equation and the boundary condition in $\bar{B} \bar{O}\left(x_{0}, r\right) \times$ $[t, t+h]$. On the contrary, in [6], $\phi$ was just a function of $x$ and the sub- or supersolution had to be built from it. This construction justified the "small time" requirement in the definition of [6]. Here $h$ is not supposed to be small. Finally we point out that the first part of condition (iii) is not restrictive at all; it is just there to avoid meaningless situations.

The next theorem which gives the relationship between the notion of generalized sub- and superflow and the level-set evolutions related to (8).

THEOREM 1.2 Suppose that the assumptions of the level-set approach hold.

(i) Let $\left(\Omega_{t}\right)_{t \in(0, T)}$ be a family of open subsets of $\bar{O}$ such that the set $\Omega:=\bigcup_{t \in(0, T)} \Omega_{t} \times\{t\}$ is open in $\bar{O} \times[0, T]$. Then $\left(\Omega_{t}\right)_{t \in(0, T)}$ is a generalized superflow with normal velocity $-F$ and angle boundary condition $G$ if and only if the function $\chi=\mathbb{1}_{\Omega}-\mathbb{1}_{\Omega^{c}}$ is a viscosity supersolution of $[8$ (i)-(ii).

(ii) Let $\left(\mathcal{F}_{t}\right)_{t \in(0, T)}$ be a family of closed subsets of $\bar{O}$ such that the set $\mathcal{F}:=\bigcup_{t \in(0, T)} \mathcal{F}_{t} \times\{t\}$ is closed in $\bar{O} \times[0, T]$. Then $\left(\mathcal{F}_{t}\right)_{t \in(0, T)}$ is a generalized subflow with normal velocity $-F$ and angle boundary condition $G$ if and only if the function $\bar{\chi}=\mathbb{1}_{\mathcal{F}}-\mathbb{1}_{\mathcal{F} c}$ is a viscosity subsolution of (8)(i)-(ii).

Proof. We only prove the result in the superflow-supersolution case, the other case being proved similarly. The proof is strongly inspired by the corresponding one in [6]; we give it in detail for the sake of completeness.

We first assume that $\chi=\mathbb{1}_{\Omega}-\mathbb{1}_{\Omega^{c}}$ is a supersolution of $(8)$ and show that $\left(\Omega_{t}\right)_{t \in(0, T)}$ is a generalized superflow. To do that, we consider a smooth function $\phi$ satisfying conditions (i)-(v) in Definition 1.1

We remark that, changing $\phi$ to $\eta \phi$ for $\eta>0$ small enough and using the fact that $F$ and $G$ satisfy respectively (A3) and (A4), we may assume without loss of generality that $\phi \leqslant 1$ in $\bar{B}_{\bar{O}}\left(x_{0}, r\right) \times[t, t+h]$.

We consider $m:=\min _{\bar{B}} \overline{\bar{O}}_{\left(x_{0}, r\right) \times[t, t+h]}(\chi-\phi)$. Since $\chi$ is lsc and $\phi$ is continuous, this minimum is attained. But, since $\chi$ is a supersolution of $(8)$ (i)-(ii) and since $\phi$ satisfies conditions (i) and (ii), it cannot be attained in $B_{O}\left(x_{0}, r\right) \times(t, t+h]$, neither in $O$ nor on $\partial O$. Therefore it has to be attained either on $\partial B \bar{o}\left(x_{0}, r\right)$ or at time $t$. 
Now we examine the consequences of (iv) and (v). For $(x, s) \in\left(\partial B \bar{O}_{0}\left(x_{0}, r\right) \times[t, t+h]\right) \cup$ $\left(\bar{B} \bar{O}\left(x_{0}, r\right) \times\{t\}\right)$, we have either

- $x \in \Omega_{s}$, then $\chi(x, s)=1$ and $(\chi-\phi)(x, s) \geqslant 0$ because $\phi \leqslant 1$ in $\bar{B} \bar{O}\left(x_{0}, r\right) \times[t, t+h]$, or

- $x \notin \Omega_{s}$, then $\chi(x, s)=-1$ and $(\chi-\phi)(x, s) \geqslant-1+\delta$ with $\delta>0$ because (iv) and (v) imply that for such points $\phi(x, s) \leqslant-\delta$. Notice that this $\delta$ can be taken uniform in $s$.

We conclude that $m \geqslant-1+\delta$ and so if $y \notin \Omega_{t+h}$, we have

$$
\chi(y, t+h)-\phi(y, t+h) \geqslant-1+\delta,
$$

which yields $\phi(y, t+h) \leqslant-\delta$ because $\chi(y, t+h)=-1$. Finally this means

$$
\left\{y \in \bar{B} \bar{O}\left(x_{0}, r\right): \phi(y, t+h) \geqslant 0\right\} \cap \Omega_{t+h}^{c}=\emptyset,
$$

which implies the desired inclusion.

Conversely, we assume that $\left(\Omega_{t}\right)_{t \in(0, T)}$ is a generalized superflow and we show that $\chi$ is a supersolution of $(8)$. Let $(x, t) \in \bar{O} \times(0, T)$ be a strict local minimum point of $\chi-\phi$ where $\phi \in C^{\infty}(\bar{O} \times[0, T])$. Changing $\phi$ to $\phi-\phi(x, t)$ if necessary, we may assume that $\phi(x, t)=0$. We consider separately the cases $(x, t) \in O \times(0, T)$ and $(x, t) \in \partial O \times(0, T)$.

If $(x, t) \in O \times(0, T)$, we have to show the inequality

$$
\frac{\partial \phi}{\partial t}(x, t)+F^{*}\left(x, t, D \phi(x, t), D^{2} \phi(x, t)\right) \geqslant 0 .
$$

This is obvious if $(x, t)$ is in the interior of either $\{\chi=1\}$ or $\{\chi=-1\}$. Indeed, in these two cases $\chi$ is constant in a neighborhood of $(x, t)$. Hence $\frac{\partial \phi}{\partial t}(x, t)=0, D \phi(x, t)=0, D^{2} \phi(x, t) \leqslant 0$ and the inequality follows since, by the local boundedness of $F$ and (A3), we have $F^{*}(x, t, 0,0)=0$.

Assume that $(x, t) \in \partial\{\chi=1\} \cap \partial\{\chi=-1\})$. The lower semicontinuity of $\chi$ yields $\chi(x, t)=$ -1 . We suppose by contradiction that, for some $\alpha>0$,

$$
\frac{\partial \phi}{\partial t}(x, t)+F^{*}\left(x, t, D \phi(x, t), D^{2} \phi(x, t)\right)<-\alpha .
$$

Since $\phi$ is smooth and $F^{*}$ is usc, we can find $r, h>0$ such that $\bar{B}(x, r) \subset O$ and for all $(y, s) \in$ $\bar{B}(x, r) \times[t-h, t]$,

$$
\frac{\partial \phi}{\partial t}(y, s)+F^{*}\left(y, s, D \phi(y, s), D^{2} \phi(y, s)\right)<-\alpha / 2
$$

Moreover, since $(x, t)$ is a strict local minimum point of $\chi-\phi$, by taking smaller $r$ and $h$ if necessary, we can assume that also, for $(y, s) \in \bar{B} \bar{O}(x, r) \times[t-h, t]$ and $(y, s) \neq(x, t)$,

$$
\chi(x, t)-\phi(x, t)=-1<\chi(y, s)-\phi(y, s) .
$$

We first consider the case when $|D \phi(x, t)| \neq 0$. For $0<\delta \ll 1$, we introduce the function $\phi_{\delta}(y, s):=\phi(y, s)+\delta(s-(t-h))$. Since $\phi(x, t)=0$ and $D \phi(x, t) \neq 0$, it is easy to see that if $h$ and $\delta$ are small enough then, for any $t-h \leqslant s \leqslant t,\left\{y \in B(x, r): \phi_{\delta}(y, s)=0\right\} \neq \emptyset$. Moreover

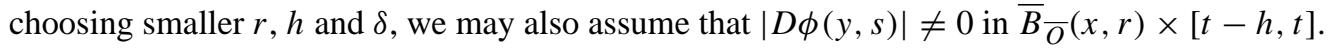


We observe that, for $\delta>0$ small enough, because of $[15]$, we have both

$$
\phi_{\delta}(y, s)-1<\chi(y, s)
$$

for all $(y, s) \in(\bar{B} \bar{O}(x, r) \times\{t-h\}) \cup(\partial B \bar{O}(x, r) \times[t-h, t])$ and

$$
\frac{\partial \phi_{\delta}}{\partial t}(y, s)+F^{*}\left(y, s, D \phi_{\delta}(y, s), D^{2} \phi_{\delta}(y, s)\right)<-\alpha / 4
$$

for all $(y, s) \in \bar{B} \bar{O}(x, r) \times[t-h, t]$.

The inequality 16 implies that

$$
\left\{y \in \bar{B} \bar{O}(x, r): \phi_{\delta}(y, t-h) \geqslant 0\right\} \subset \Omega_{t-h},
$$

and for all $s \in[t-h, t]$,

$$
\left\{y \in \partial \bar{B} \bar{O}(x, r): \phi_{\delta}(y, s) \geqslant 0\right\} \subset \Omega_{s} .
$$

By the definition of superflow, using the fact that condition (ii) is empty since $\bar{B}(x, r) \cap \partial O=\emptyset$, this yields

$$
\left\{y \in O: \phi_{\delta}(y, t)>0\right\} \cap \bar{B}(x, r) \subset \Omega_{t} .
$$

But, since $\phi_{\delta}(x, t)=\delta h>0$, we deduce that $x \in \Omega_{t}$, and this is a contradiction.

Now we turn to the case when $|D \phi(x, t)|=0$. We can assume without loss of generality that $D^{2} \phi(x, t)=0$ as well (see e.g. [4]) and we have to show that $\frac{\partial \phi}{\partial t}(x, t) \geqslant 0$.

Suppose by contradiction that $a:=\frac{\partial \phi}{\partial t}(x, t)<0$. Since $\phi(x, t)=0$, we have

$$
\phi(y, s)=\frac{\partial \phi}{\partial t}(x, t)(s-t)+o(|s-t|)+o\left(|y-x|^{2}\right) \quad \text { as } s \rightarrow t,|y-x| \rightarrow 0 .
$$

Thus, for all $\varepsilon>0$, there exist $r, h>0$ such that

$$
\phi(y, s) \geqslant \frac{a}{2}(s-t)-\varepsilon|y-x|^{2} \quad \text { for all }(y, s) \in \bar{B} \bar{O}(x, r) \times[t-h, t]
$$

and $\bar{B} \bar{O}(x, r) \cap \partial O=\emptyset$. We take $\beta>0$ small enough such that

$$
\beta+\phi(y, s)-1<\chi(y, s)
$$

for all $(y, s) \in(\bar{B} \bar{O}(x, r) \times\{t-h\}) \cup(\partial B \bar{O}(x, r) \times[t-h, t])$. By taking $h$ smaller we may also suppose that $\beta>-(a / 2) h$.

Then we consider the function $\psi_{\beta}(y, s)=(a / 2)(s-t)-\varepsilon|y-x|^{2}+\beta$. Since $F^{*}$ is upper semicontinuous and $F^{*}(y, s, 0,0)=0$ for any $y$ and $s$, for small $\varepsilon$ we have

$$
\frac{a}{2}+F^{*}(y, s,-2 \varepsilon(y-x),-2 \varepsilon I)<0 \quad \text { on } \bar{B} \bar{O}(x, r) \times[t-h, t] .
$$

Examining the function $\psi_{\beta}$ and choosing perhaps smaller $\beta$ and $h$, one easily sees that $\{y \in$ $\left.B(x, r): \psi_{\beta}(y, s)=0\right\} \neq \varnothing$ for any $t-h \leqslant s \leqslant t$. Moreover $\left|D \psi_{\beta}(y, s)\right| \neq 0$ in $\left\{(y, s) \in \bar{B} \bar{O}(x, r) \times[t-h, t]: \psi_{\beta}(y, s)=0\right\}$ and

$$
\left\{y \in \bar{B} \bar{O}(x, r): \psi_{\beta}(y, t-h) \geqslant 0\right\} \subseteq \Omega_{t-h},
$$


and for all $s \in[t-h, t]$,

$$
\left\{y \in \partial B_{\bar{O}}(x, r): \psi_{\beta}(y, s) \geqslant 0\right\} \subseteq \Omega_{s} .
$$

Thus, since $\left(\Omega_{t}\right)_{t}$ is a generalized superflow, we have

$$
\left\{y \in B_{\bar{O}}(x, r): \psi_{\beta}(y, t)>0\right\} \subset \Omega_{t} .
$$

But again $\psi_{\beta}(x, t)=\beta>0$, and this means $x \in \Omega_{t}$, which is a contradiction.

Now we examine the case $(x, t) \in \partial O \times(0, T)$ and suppose by contradiction that

$$
G(x, t, D \phi(x, t))<0 \quad \text { and } \quad \frac{\partial \phi}{\partial t}(x, t)+F^{*}\left(x, t, D \phi(x, t), D^{2} \phi(x, t)\right)<0 .
$$

We note that the first strict inequality implies that $D \phi(x, t) \neq 0$. Thus we can argue exactly as in the first case above by defining the function $\phi_{\delta}$. Indeed, we observe that $D \phi_{\delta}(x, t)=D \phi(x, t)$ and we can choose $r, h>0$ small enough such that

$$
G\left(y, s, D \phi_{\delta}(y, s)\right)<0 \quad \text { in }(\partial O \cap \bar{B}(x, r)) \times[t-h, t],
$$

and

$$
\frac{\partial \phi_{\delta}}{\partial t}+F^{*}\left(y, s, D \phi_{\delta}(y, s), D^{2} \phi_{\delta}(y, s)\right)<0 \quad \text { in } \bar{B} \bar{O}(x, r) \times[t-h, t] .
$$

Thus the proof is complete.

\section{Applications to the asymptotics of reaction-diffusion equations}

\subsection{The abstract method}

In this section, we present an abstract method to study the asymptotics of solutions to semilinear reaction-diffusion equations in bounded domains with Neumann boundary conditions. We essentially follow the ideas of [6] but, because of the particularities of our definition and especially (v) in Definition 1.1, we have to modify this abstract method slightly.

In the asymptotic problems we have in mind, we are given a family $\left(u_{\varepsilon}\right)_{\varepsilon}$ of bounded functions on $\bar{O} \times[0, T]$, typically the solutions of reaction-diffusion equations with Neumann type boundary conditions and with a small parameter $\varepsilon>0$. The aim is to show that there exists a generalized flow $\left(\Omega_{t}\right)_{t \in(0, T]}$ on $\bar{O}$ with a certain normal velocity and angle boundary on $\partial O$ such that, as $\varepsilon \rightarrow 0$,

$$
\begin{array}{ll}
u_{\varepsilon}(x, t) \rightarrow b(x, t) & \text { if }(x, t) \in \Omega=\bigcup_{t \in(0, T)} \Omega_{t} \times\{t\}, \\
u_{\varepsilon}(x, t) \rightarrow a(x, t) & \text { if }(x, t) \in \bar{\Omega}^{c},
\end{array}
$$

where, for all $(x, t), a(x, t), b(x, t) \in \mathbb{R}$ can be interpreted as local equilibria of this system.

Unfortunately, although the method we are going to use is very close in spirit to the one of [6], we cannot present it in a framework as general as in [6]. This is due to the fact that our method relies on more local arguments, which, on the other hand, can be seen as an advantage of it.

In order to be more specific and to present the main steps of the method, we first assume that there exist sequences $\left(a_{\varepsilon}\right)_{\varepsilon}$ and $\left(b_{\varepsilon}\right)_{\varepsilon}$ of real-valued functions defined in $\bar{O} \times[0, T]$ such that

$$
a_{\varepsilon}(x, t) \leqslant u_{\varepsilon}(x, t) \leqslant b_{\varepsilon}(x, t) \quad \text { in } \bar{O} \times[0, T],
$$

and $a_{\varepsilon} \rightarrow a, b_{\varepsilon} \rightarrow b$ uniformly in $\bar{O} \times[0, T]$ as $\varepsilon \rightarrow 0$. 
We recall the definition of half-relaxed limits in the theory of viscosity solutions: if $z_{\varepsilon}: \bar{O} \times$ $[0, T] \rightarrow \mathbb{R}$ is a sequence of functions, we set

$$
\limsup z_{\varepsilon} z_{\varepsilon}(x, t):=\limsup _{\substack{(y, s) \rightarrow(x, t) \\ \varepsilon \rightarrow 0}} z_{\varepsilon}(y, s), \quad \liminf _{*} z_{\varepsilon}(x, t):=\liminf _{\substack{(y, s) \rightarrow(x, t) \\ \varepsilon \rightarrow 0}} z_{\varepsilon}(y, s) .
$$

Our method consists in introducing, for some well chosen $\tau \geqslant 0$, the sets

$$
\begin{aligned}
& \Omega^{1}=\operatorname{Int}\left\{(x, t) \in \bar{O} \times[0, T]: \liminf _{*}\left[\frac{u_{\varepsilon}-b_{\varepsilon}}{\varepsilon^{\tau}}\right](x, t) \geqslant 0\right\}, \\
& \Omega^{2}=\operatorname{Int}\left\{(x, t) \in \bar{O} \times[0, T]: \limsup \sup ^{*}\left[\frac{u_{\varepsilon}-a_{\varepsilon}}{\varepsilon^{\tau}}\right](x, t) \leqslant 0\right\} .
\end{aligned}
$$

Then we are going to consider the families $\left(\Omega_{t}^{1}\right)_{t}$ and $\left(\Omega_{t}^{2}\right)_{t}$ defined by

$$
\begin{aligned}
& \Omega_{t}^{1}=\Omega^{1} \cap(\bar{O} \times\{t\}), \\
& \Omega_{t}^{2}=\Omega^{2} \cap(\bar{O} \times\{t\}) .
\end{aligned}
$$

For simplicity of notations, for $i=1,2$, we identify $\Omega_{t}^{i}$ and $\left(\Omega_{t}^{i}\right)^{c}$ with their projections in $\bar{O}$.

It is worth noticing that $\Omega^{1}, \Omega^{2}$ are defined as subsets of $\bar{O} \times(0, T]$, they are open by definition and disjoint. In particular, in view of Theorem 1.2, we remark that by construction the functions $\chi=\mathbb{1}_{\Omega^{1}}-\mathbb{1}_{\left(\Omega^{1}\right)^{c}}$ and $\bar{\chi}=\mathbb{1}_{\left(\Omega^{2}\right)^{c}}-\mathbb{1}_{\Omega^{2}}$ are respectively lower and upper semicontinuous on $\bar{O} \times(0, T]$; here, in fact, $\Omega^{1}$ has to be read as $\bigcup_{t \in(0, T]} \Omega_{t}^{1} \times\{t\}$ and $\Omega^{2}$ as $\bigcup_{t \in(0, T]} \Omega_{t}^{2} \times\{t\}$. We finally point out that $\chi, \bar{\chi}$ can be extended by lower or upper semicontinuity to $\bar{O} \times[0, T]$, and below we keep the same notations for these extensions.

We come back below to the role of $\tau$ which will be clear (at least we hope so!) in the examples we will treat, where we mainly use either $\tau=1$ or $\tau=0$.

Our method can be described in three steps.

1. Initialization: we have to determine the traces $\Omega_{0}^{1}$ and $\Omega_{0}^{2}$ of $\Omega^{1}$ and $\Omega^{2}$ for $t=0$. A convenient way to define these traces is through the functions $\chi$ and $\bar{\chi}$ :

$$
\Omega_{0}^{1}=\{x \in \bar{O}: \chi(x, 0)=1\}, \quad \Omega_{0}^{2}=\{x \in \bar{O}: \bar{\chi}(x, 0)=-1\} .
$$

2. Propagation: we have to show that $\left(\Omega_{t}^{1}\right)_{t}$ and $\left(\left(\Omega_{t}^{2}\right)^{c}\right)_{t}$ are respectively super- and subflows with normal velocity $-F$ and angle condition $G$.

3. Conclusion: we use the following result for the above $\left(\Omega_{t}^{1}\right)_{t}$ and $\left(\left(\Omega_{t}^{2}\right)^{c}\right)_{t}$; its proof is a consequence of Theorems 1.1 and 1.2 and therefore we omit it.

COROLlary 2.1 Assume that the assumptions of the level-set approach hold and that the above families $\left(\Omega_{t}^{1}\right)_{t}$ and $\left(\left(\Omega_{t}^{2}\right)^{c}\right)_{t}$ are respectively super- and subflows with normal velocity $-F$ and angle boundary condition $G$ and suppose there exists $\left(\partial \Omega_{0}^{1}, \Omega_{0}^{+}, \Omega_{0}^{-}\right) \in \mathcal{E}$ such that $\Omega_{0}^{+} \subseteq \Omega_{0}^{1}$ and $\Omega_{0}^{-} \subseteq \Omega_{0}^{2}$. Then if $\left(\Gamma_{t}, \Omega_{t}^{+}, \Omega_{t}^{-}\right)$is the level-set evolution of $\left(\partial \Omega_{0}^{1}, \Omega_{0}^{+}, \Omega_{0}^{-}\right)$we have:

(i) for all $t \in[0, T]$,

$$
\Omega_{t}^{+} \subset \Omega_{t}^{1} \subset \Omega_{t}^{+} \cup \Gamma_{t}, \quad \Omega_{t}^{-} \subset \Omega_{t}^{2} \subset \Omega_{t}^{-} \cup \Gamma_{t},
$$


(ii) if $\bigcup_{t} \Gamma_{t} \times\{t\}$ satisfies the no-interior condition, then for all $t \in[0, T]$, we have

$$
\Omega_{t}^{+}=\Omega_{t}^{1}, \quad \Omega_{t}^{-}=\Omega_{t}^{2}
$$

We now comment on the first two steps of our method. It is first worth pointing out that compared with [6], we have a different definition of the families $\left(\Omega_{t}^{1}\right)_{t}$ and $\left(\Omega_{t}^{2}\right)_{t}:$ in [6] they were defined IN ANY CASE as

$$
\begin{aligned}
& \Omega^{1}=\left\{x: \liminf _{*} u_{\varepsilon}(x, t)=b(x, t)\right\}, \\
& \Omega^{2}=\left\{x: \lim \sup ^{*} u_{\varepsilon}(x, t)=a(x, t)\right\} .
\end{aligned}
$$

We are led to introduce the parameter $\tau$ in the definition of the families $\left(\Omega_{t}^{1}\right)_{t}$ and $\left(\Omega_{t}^{2}\right)_{t}$ because of condition (v) in Definition 1.1, and for example in the study of the asymptotics of the Allen-Cahn equation when the normal velocity is mean curvature (see the next section), it will be natural to work with $\tau=1$.

A technical consequence of our definition of the families $\left(\Omega_{t}^{1}\right)_{t}$ and $\left(\Omega_{t}^{2}\right)_{t}$ is that the initialization of the front will be done only at time $t=0$, whereas in [6] it has to be done at any time. We also mention that this initialization procedure at time $t=0$ consists in constructing globally in $\bar{O}$ sub- and supersolutions of the $u_{\varepsilon}$-equation, but—and this will simplify matters—-these sub- and supersolutions will be associated with radially symmetric moving fronts.

The second step will consist, as in [6], in constructing suitable smooth sub- and supersolutions to the Neumann problem satisfied by $u_{\varepsilon}$, but with two main differences: we build them only locally, i.e. in balls $\bar{B} \bar{O}(x, r)$, where $x \in \bar{O}$, with Neumann boundary conditions on $B(x, r) \cap \partial O$ if this set is not empty and Dirichlet boundary conditions on $\partial B \bar{O}(x, r)$. In contrast to [6], this construction will NOT be local in time since it will be done in time intervals of the form $[t, t+h]$ where $h$ is not supposed to be small; moreover, and this is also a difference with [6], the comparison of $u_{\varepsilon}$ and these sub- and supersolutions will be done on $\bar{B} \bar{O}(x, r) \times[t, t+h]$ and not in $\bar{O} \times[t, t+h]$. This is the reason why we are not able to describe the method in the same abstract way as in [6].

\subsection{The Allen-Cahn equation}

This section is devoted to the study of the model case of the Allen-Cahn equation in a bounded domain with a Neumann type boundary condition, which will also be the opportunity to give the reader a more precise idea of how the abstract method works. More precisely we focus our attention on the following initial boundary value problem:

$$
\begin{cases}u_{\varepsilon, t}-\Delta u_{\varepsilon}+\varepsilon^{-2} f\left(u_{\varepsilon}\right)=0 & \text { in } O \times(0, \infty), \\ G\left(x, t, D u_{\varepsilon}\right)=0 & \text { on } \partial \times(0, \infty), \\ u_{\varepsilon}=g & \text { on } \bar{O} \times\{0\},\end{cases}
$$

where $g$ is a real-valued continuous function in $\bar{O}$ and $G$ satisfies the assumptions of the level-set approach and in particular (A2) and (A4). Concerning the reaction term $f: \mathbb{R} \rightarrow \mathbb{R}$, throughout the paper, we assume that

$$
\left\{\begin{array}{l}
f \in C^{2}(\mathbb{R}) \text { has exactly three zeros } m_{-}<m_{0}<m_{+}, \\
f(s)>0 \text { in }\left(m_{-}, m_{0}\right) \text { and } f(s)<0 \text { in }\left(m_{0}, m_{+}\right), \\
f^{\prime}\left(m_{ \pm}\right)>0, f^{\prime \prime}\left(m_{-}\right)<0 \text { and } f^{\prime \prime}\left(m_{+}\right)>0 .
\end{array}\right.
$$


We also assume that the equation admits, for each $e \in S^{N-1}$, traveling wave solutions connecting $m_{-}$and $m_{+}$, i.e., solutions of the form

$$
u(x, t)=q(x \cdot e-c t),
$$

where $q: \mathbb{R} \rightarrow \mathbb{R}$ is such that $q( \pm \infty)=m_{ \pm}$. Indeed, we assume that

$$
\left\{\begin{array}{l}
\text { there exists a unique pair }(c, q) \text { such that } \\
c \dot{q}+\ddot{q}=f(q) \text { on } \mathbb{R}, \quad \dot{q}>0 \text { on } \mathbb{R}, \quad q(0)=m_{0}, \\
q(s) \rightarrow m_{ \pm}, \text {exponentially fast, as } s \rightarrow \pm \infty .
\end{array}\right.
$$

The existence and properties of such pairs $(c, q)$ are studied, for example, in Aronson and Weinberger [2], to which we refer for details.

In the case where the wells of the potential $W: \mathbb{R} \rightarrow \mathbb{R}$, defined by $W^{\prime}=f$, have the same depth, i.e.,

$$
W\left(m_{+}\right)-W\left(m_{-}\right)=0,
$$

it follows that $c=0$ in (25) and $q$ solves

$$
\ddot{q}=f(q) \quad \text { in } \mathbb{R} .
$$

In the case of linear, homogeneous Neumann boundary conditions, the asymptotics of (23) was first studied by Chen [7] under the assumption that the resulting interface is smooth (i.e. essentially for smooth initial interface and for small time) and by Katsoulakis, Kossioris and Reitich [17] globally in time but for convex domains $O$.

The front evolution associated with the asymptotics of (23) is a motion by mean curvature with Neumann boundary conditions. The corresponding geometric pde is

$$
\begin{cases}u_{t}-\operatorname{tr}\left[(I-\widehat{D u} \otimes \widehat{D u}) D^{2} u\right]=0 & \text { in } O \times(0, \infty), \\ G(x, t, D u)=0 & \text { on } \partial O \times(0, \infty), \\ u=u_{0} & \text { on } \bar{O} \times\{0\},\end{cases}
$$

where $\hat{p}=p /|p|$ for $p \in \mathbb{R}^{N} \backslash\{0\}$.

The main result is

THEOREM 2.1 Assume that $G$ satisfies the assumptions of the level-set approach and that (24), 25), 26) hold. Let $u_{\varepsilon}$ be the solution of 23 , where $g: \bar{O} \rightarrow\left[m_{-}, m_{+}\right]$is a continuous function such that the set $\Gamma_{0}=\left\{x: g(x)=m_{0}\right\}$ is a nonempty subset of $O$. Then, as $\varepsilon \rightarrow 0$,

$$
u_{\varepsilon}(x, t) \rightarrow \begin{cases}m_{+} & \{u>0\}, \\ m_{-} & \text {locally uniformly in } \\ & \{u<0\},\end{cases}
$$

where $u$ is the unique viscosity solution of (28) with $u_{0}=d_{0}$, the signed distance to $\Gamma_{0}$, which is positive in the set $\left\{g>m_{0}\right\}$ and negative in $\left\{g<m_{0}\right\}$. If, in addition, the no-interior condition (13) holds, then, as $\varepsilon \rightarrow 0$,

$$
u_{\varepsilon}(x, t) \rightarrow \begin{cases}m_{+} & \text {locally uniformly in } \\ m_{-} & \frac{\{u>0\},}{\{u>0\}} .\end{cases}
$$


Proof. We consider the families $\left(\Omega_{t}^{1}\right)_{t}$ and $\left(\Omega_{t}^{2}\right)_{t}$ of sets defined in Section 2.1 by 18, , 19 with $b_{\varepsilon}, b \equiv m_{+}, a_{\varepsilon}, a \equiv m_{-}$and with $\tau=1$, and let $\Omega_{0}^{1}, \Omega_{0}^{2}$ be defined by 22 .

The proof of Theorem 2.1 follows the abstract method described in Section 2.1 and consists of two main steps.

The first step (Proposition 2.1) consists in showing that $\left\{x \in \bar{O}: g(x)>m_{0}\right\} \subseteq \Omega_{0}^{1}$ and $\left\{x \in \bar{O}: g(x)<m_{0}\right\} \subseteq \Omega_{0}^{2}$. The second step (Proposition 2.2 is devoted to verifying that the families $\left(\Omega_{t}^{1}\right)_{t>0}$ and $\left(\left(\Omega_{t}^{2}\right)^{c}\right)_{t>0}$ are respectively a generalized superflow and subflow with normal velocity $-F\left(D d, D^{2} d\right)=-\Delta d$ and angle condition $G$. Once these two steps are performed, the conclusion follows easily from Corollary 2.1 .

We will give the proof of the two steps described in the proof of Theorem 2.1, we will do that only for the $\Omega^{1}$-case, the $\Omega^{2}$-case being obtained by similar arguments.

We first point out a key property of $G$ which is used in what follows to check the Neumann boundary condition. To formulate it, we use the following notation: for $p \in \mathbb{R}^{N}$ and $x \in \partial O$, $\mathcal{T}(p):=p-(p \cdot n(x)) n(x)$, so $\mathcal{T}(p)$ represents the projection of $p$ on the tangent hyperplane to $\partial O$ at $x$.

LEMma 2.1 Assume that (A2) and (A4) hold and that, for some $x \in \partial O, t \in(0, T)$ and $\tilde{p} \in \mathbb{R}^{N}$, we have $G(x, t, \tilde{p}) \leqslant 0$ (resp. $G(x, t, \tilde{p}) \geqslant 0)$. Then there exists a constant $K(T)$ such that if $p \cdot n(x) \leqslant-K(T)|\mathcal{T}(p)|$, then

$$
G(x, t, \tilde{p}+p) \leqslant 0
$$

(resp. if $p \cdot n(x) \geqslant K(T)|\mathcal{T}(p)|$, then $G(x, t, \tilde{p}+p) \geqslant 0$.)

Before providing the very short proof of Lemma 2.1, we remark that, by (A4), $G(x, t, 0) \equiv 0$ and therefore the above result applies to $\tilde{p}=0$.

Proof of Lemma 2.1. We prove only the first inequality. We set $\lambda:=-p \cdot n(x)$; we may assume it to be positive. Since $p=\mathcal{T}(p)-\lambda n(x)$ by definition we have

$$
G(x, t, \tilde{p}+p)=G(x, t, \tilde{p}+\mathcal{T}(p)-\lambda n(x)) .
$$

By (A2) we have

$$
G(x, t, \tilde{p}+\mathcal{T}(p)-\lambda n(x)) \leqslant G(x, t, \tilde{p}+\mathcal{T}(p))-v(T) \lambda .
$$

Since, by (A2), there is a modulus of continuity $m$ of $G$ in $p$ (which is uniform with respect to $x$ and $t$ ), we see, by (A4), that

$$
\begin{aligned}
G(x, t, \tilde{p}+\mathcal{T}(p)) & =\lambda G\left(x, t, \lambda^{-1}(\tilde{p}+\mathcal{T}(p))\right) \\
& \leqslant \lambda\left[G\left(x, t, \lambda^{-1} \tilde{p}\right)+m\left(\lambda^{-1}|\mathcal{T}(p)|\right)\right] \\
& =G(x, t, \tilde{p})+\lambda m\left(\lambda^{-1}|\mathcal{T}(p)|\right) \\
& \leqslant \lambda m\left(\lambda^{-1}|\mathcal{T}(p)|\right) .
\end{aligned}
$$

Combining 29], 30] and (31) we obtain

$$
G(x, t, \tilde{p}+p) \leqslant \lambda\left[m\left(\lambda^{-1}|\mathcal{T}(p)|\right)-v(T)\right] .
$$

To conclude, it suffices to show that the expression in square brackets is negative if $\lambda$ is large enough compared to $\mathcal{T}(p)$, which is obviously the case since $m(r) \rightarrow 0$ as $r \downarrow 0^{+}$. 
Now we present the consecutive steps of the proof of Theorem 2.1

STEP 1: Initialization. We have

Proposition 2.1 The set $\left\{x \in \bar{O}: g(x)>m_{0}\right\}$ is contained in $\Omega_{0}^{1}$.

Proof. Let $x_{0} \in\left\{x \in \bar{O}: g(x)>m_{0}\right\}$. We have to show that $x_{0} \in \Omega_{0}^{1}$. We only consider the case $x_{0} \in \partial O \cap\left\{g(x)>m_{0}\right\}$, the case $x_{0} \in O \cap\left\{g(x)>m_{0}\right\}$ being similar and even simpler.

Let $r>0$ be such that $g(y)>m_{0}$ for all $y \in \bar{B} \bar{O}\left(x_{0}, r\right)$. By the smoothness of $O$, if $\eta>0$ is small enough and if $\bar{x}:=x_{0}-\eta n\left(x_{0}\right)$ then $B(\bar{x}, \eta) \subseteq O$ and $\bar{B}(\bar{x}, \eta) \cap \partial O=\left\{x_{0}\right\}$. Consider the function

$$
\phi_{\eta}(x)=\eta^{2}-|x-\bar{x}|^{2} .
$$

We observe that $D \phi_{\eta}\left(x_{0}\right) \cdot n\left(x_{0}\right)=-2 \eta<0$.

Thus we can find $R>\eta$ and $\bar{\delta}>0$ such that $B(\bar{x}, R) \subseteq B\left(x_{0}, r\right)$ and the function $\phi(x)=$ $R^{2}-|x-\bar{x}|^{2}$ satisfies $D \phi(x) \cdot n(x)<0$ on $\{x \in \partial O:|d(x)|<\bar{\delta}\}, d(\cdot)$ being the signed-distance function to the set $\{x: \phi(x)=0\}$. By Lemma 2.1 choosing $R$ close enough to $\eta$, we may also have $G(x, t, D \phi(x))<0$ on $\{x \in \partial O:|d(x)|<\bar{\delta}\}$ for, say, any $t \leqslant 1$.

By the choice of $R$, there is $0<\delta^{\prime}<\frac{1}{2}\left(m_{+}-m_{0}\right) \wedge \bar{\delta}$ such that for all $0<\delta<\delta^{\prime}$ we have

$$
\left.u_{\varepsilon}(x, 0) \geqslant\left(m_{0}+2 \delta\right) \mathbb{1}_{\bar{B}}\left(x_{0}, r\right)+m_{-} \mathbb{1}_{(\bar{B}} \bar{O}\left(x_{0}, r\right)\right)^{c} \geqslant\left(m_{0}+\delta\right) \mathbb{1}_{\{\phi>0\}}+m_{-} \mathbb{1}_{\{\phi \leqslant 0\}} \quad \text { in } \bar{O} .
$$

We introduce the function $\Phi: \bar{O} \times[0, T] \rightarrow \mathbb{R}$ given by

$$
\Phi(x, t)=\phi(x)-C t,
$$

with $C>0$ to be chosen later, and denote by $d(\cdot, t)$ the signed distance to the set $\{\Phi(\cdot, t)=0\}$ which is defined so as to have the same sign as $\Phi$ in $\bar{O} \times[0, T]$. Here

$$
d(x, t)=\left[\left(R^{2}-C t\right)^{+}\right]^{1 / 2}-|x-\bar{x}| .
$$

Now we need the following two lemmas:

LEMMA 2.2 (Very small time initialization) Under the assumptions of Theorem 2.1 for any $\beta>0$, there are constants $\tau>0$ and $\bar{\varepsilon}>0$ (depending on $\beta$ ) such that, for all $0<\varepsilon \leqslant \bar{\varepsilon}$, we have

$$
u_{\varepsilon}\left(x, t_{\varepsilon}\right) \geqslant\left(m_{+}-\beta \varepsilon\right) \mathbb{1}_{\{d(x, 0) \geqslant \beta\}}+m_{-} \mathbb{1}_{\{d(x, 0)<\beta\}} \quad \text { on } \bar{O},
$$

where $t_{\varepsilon}=\tau \varepsilon^{2}|\log \varepsilon|$

LEMMA 2.3 (Propagation I: global version) There exist $\bar{h}>0, \bar{\beta}>0$, depending only on the function $\phi$ defined in 32 , such that if $\beta \leqslant \bar{\beta}(\phi)$ and $\varepsilon \leqslant \bar{\varepsilon}(\beta, \phi)$, then there exists a subsolution $w^{\varepsilon, \beta}$ of 23 in $\bar{O} \times(0, h)$ such that

$$
w^{\varepsilon, \beta}(\cdot, 0) \leqslant\left(m_{+}-\beta \varepsilon\right) \mathbb{1}_{\{d(x, 0) \geqslant \beta\}}+m_{-} \mathbb{1}_{\{d(x, 0)<\beta\}} \quad \text { on } \bar{O} .
$$

Moreover, if $(x, t) \in B \bar{O}\left(x_{0}, r\right) \times(0, \bar{h})$ satisfies $d(x, t)>2 \beta$, then

$$
\liminf _{*}\left[\frac{w^{\varepsilon, \beta}-m_{+}}{\varepsilon}\right](x, t) \geqslant-2 \beta .
$$


We postpone the proof of Lemmas 2.2 and 2.3 and we continue with the proof of Proposition 2.1. Lemma 2.3 yields a subsolution $w^{\varepsilon, \beta}$ of 23 such that

$$
w^{\varepsilon, \beta}(\cdot, 0) \leqslant u_{\varepsilon}\left(\cdot, t_{\varepsilon}\right) \quad \text { in } \bar{O}
$$

thus, by the maximum principle, we have

$$
w^{\varepsilon, \beta}(x, s) \leqslant u_{\varepsilon}\left(x, s+t_{\varepsilon}\right) \quad \text { on } \bar{O} \times[0, \bar{h}] .
$$

It follows that if $t \in(0, \bar{h}), x \in B_{\bar{O}}\left(x_{0}, r\right)$ and $d(x, t)>2 \beta$, then

$$
\liminf *\left[\frac{u_{\varepsilon}-m_{+}}{\varepsilon}\right](x, t) \geqslant-2 \beta .
$$

Since $\beta$ is arbitrary and does not depend on $\bar{h}$, we have $(x, t) \in \Omega_{t}^{1} \times\{t\}$ if $d(x, t)>0$ and $t>0$. According to the definition of $d$, it follows that, for $\bar{\eta}, \bar{t}>0$ small enough, $B \bar{O}\left(x_{0}, \bar{\eta}\right) \subset\{x$ : $d(x, t)>0\}$ for any $0<t<\bar{t}$. This implies that $B \bar{O}\left(x_{0}, \bar{\eta}\right) \subset \Omega_{t}^{1}$ for any $0<t<\bar{t}$ and therefore $x_{0} \in \Omega_{0}^{1}$.

Proof of Lemma 2.2. We follow (and slightly simplify) the proof of Chen [7].

1. We consider $0<\delta<\delta^{\prime}$. We are going to modify the function $f$ in two steps; we first introduce a smooth cut-off function $\zeta_{1} \in C_{0}^{\infty}(\mathbb{R})$ such that $0 \leqslant \zeta_{1} \leqslant 1$ in $\mathbb{R}, \zeta_{1}(s)=0$ in $\left(-\infty, m_{0}-\delta\right] \cup$ $\left[m_{0}+\delta, \infty\right)$ and $\zeta_{1}(s)=1$ in $\left[m_{0}, m_{0}+3 \delta / 4\right]$. We set

$$
\tilde{f}_{\delta}(s)=\left(1-\zeta_{1}(s)\right) f(s)+\zeta_{1}(s) f(s-\delta / 2) \text {. }
$$

Using the assumptions on $f$, it is easy to see that, for $\delta$ small enough, $\tilde{f}_{\delta}$ is $C^{2}$ and has exactly three zeros, $m_{-}, m_{0}+\delta / 2, m_{+} ;$moreover $\tilde{f}_{\delta} \geqslant f$ in $\mathbb{R}$ with $\tilde{f}_{\delta}(s)=f(s)$ in $\left(-\infty, m_{0}-\delta\right] \cup\left[m_{0}+\delta, \infty\right)$.

2. Then we consider another cut-off function $\zeta_{2} \in C_{0}^{\infty}(\mathbb{R})$ such that $0 \leqslant \zeta_{2} \leqslant 1$ in $\mathbb{R}, \zeta_{2}(s)=0$ in $\left(-\infty, m_{0}\right] \cup\left[m_{0}+\delta, \infty\right)$ and $\zeta_{2}(s)=1$ in $\left[m_{0}+\delta / 4, m_{0}+3 \delta / 4\right]$. Finally we consider

$$
\bar{f}_{\delta}(s)=\left(1-\zeta_{2}(s)\right) \tilde{f}_{\delta}(s)+\zeta_{2}(s) \frac{\delta / 2+m_{0}-s}{|\log \varepsilon|} .
$$

We note that, again, $\bar{f}_{\delta}$ has exactly three zeros: $m_{-}, m_{0}+\delta / 2, m_{+}$, and $\bar{f}_{\delta} \geqslant f$ in $\mathbb{R}$ with

$$
\bar{f}_{\delta}(s)=\frac{\delta / 2+m_{0}-s}{|\log \varepsilon|} \quad \text { on }\left[m_{0}+\delta / 4, m_{0}+3 \delta / 4\right] \text {. }
$$

3. Standard arguments from the theory of ordinary differential equations and 24) yield the existence of a unique solution $\chi \in C^{2}(\mathbb{R} \times[0, \infty))$ of

$$
\dot{\chi}(\xi, s)+\bar{f}_{\delta}(\chi(\xi, s))=0 \quad \text { in }[0, \infty) \text { with } \chi(\xi, 0)=\xi \in \mathbb{R}
$$

satisfying, in addition,

$$
\chi_{\xi}(\xi, s)>0 \quad \text { in } \mathbb{R} \times[0, \infty) .
$$


Moreover, one can prove that

$$
\left\{\begin{array}{l}
\text { for all } \beta>0, \text { there exists } a(\beta, \delta)>0 \text { such that } \\
\chi(\xi, s) \geqslant m_{+}-\beta \varepsilon \quad \text { for } s \geqslant a|\log \varepsilon| \text { and } \xi \geqslant \delta+m_{0},
\end{array}\right.
$$

and

$$
\left\{\begin{array}{l}
\text { for every } a>0, \text { there exists } M(a) \in \mathbb{R} \text { such that, for } \varepsilon \text { small enough, } \\
\left(\chi_{\xi}(\xi, s)\right)^{-1}|\chi \xi \xi(\xi, s)| \leqslant \varepsilon^{-1} M(a) \quad \text { for } 0<s \leqslant a|\log \varepsilon| .
\end{array}\right.
$$

It is worth mentioning that all our modifications of $f$ were done in order to have the above properties for $\chi$ (cf. Chen [7]).

4. Let $\psi$ be a nondecreasing smooth function such that $\psi^{\prime}>0$ in $\mathbb{R}$ and

$$
m_{-} \leqslant \psi \leqslant m_{0}+2 \delta \quad \text { in } \mathbb{R}, \quad \psi(z)= \begin{cases}m_{-} & \text {in }\{z<0\} \\ m_{0}+2 \delta & \text { in }\{z \geqslant \delta\}\end{cases}
$$

It is clear that

$$
\psi(d(x, 0)) \leqslant\left(m_{0}+2 \delta\right) \mathbb{1}_{\{d(x, 0) \geqslant \delta\}}+m_{-} \mathbb{1}_{\{d(x, 0)<\delta\}} \quad \text { in } \bar{O} .
$$

We define $\bar{w}: \bar{O} \times[0, \infty) \rightarrow \mathbb{R}$ by

$$
\bar{w}(x, t)=\chi\left(\psi(d(x, 0))-\varepsilon^{-1} K t, \varepsilon^{-2} t\right) .
$$

Following the computations of [7] (see also [6]), one can show that $\bar{w}$ satisfies 23)(i)-(ii) in $O \times$ $\left(0, a \varepsilon^{2}|\log \varepsilon|\right)$. As far as the Neumann boundary condition is concerned, we observe that

$$
D \bar{w}(x, t)=\chi \xi \psi^{\prime} D d(x, 0) .
$$

But, by the definition of $\psi, \psi^{\prime} \neq 0$ only if $0<d(x, 0)<\delta$. In this set by construction we have

$$
D d(x, 0) \cdot n(x)=\frac{D \phi(x)}{|D \phi(x)|} \cdot n(x)<0 .
$$

Thus since $\chi_{\xi}>0, \psi^{\prime}>0$ we have $D \bar{w}(x, t) \cdot n(x)<0$ and so $G(x, t, D \bar{w}) \leqslant 0$ by (A4) and Lemma 2.1. Furthermore,

$$
\bar{w}(x, 0) \leqslant\left(m_{0}+2 \delta\right) \mathbb{1}_{\{d(x, 0) \geqslant \delta\}}+m_{-} \mathbb{1}_{\{d(x, 0)<\delta\}} \leqslant u_{\varepsilon}(x, 0) \quad \text { on } \bar{O} .
$$

Thus the maximum principle yields

$$
\bar{w}(x, s) \leqslant u_{\varepsilon}(x, s) \quad \text { in } \bar{O} \times\left[0, t_{\varepsilon}\right]
$$

5. Evaluating 39 for $t=a \varepsilon^{2}|\log (\varepsilon)|$ and for $x$ such that $d(x, 0) \geqslant \delta$ yields

$$
\chi\left(m_{0}+2 \delta-K a \varepsilon|\log \varepsilon|, a|\log \varepsilon|\right) \leqslant u_{\varepsilon}\left(x, a \varepsilon^{2}|\log \varepsilon|\right) .
$$

But since for $\varepsilon$ small enough

$$
m_{0}+2 \delta-K a \varepsilon|\log \varepsilon| \geqslant m_{0}+\delta
$$


it follows from $(36)$ that

$$
m_{+}-\beta \varepsilon \leqslant u_{\varepsilon}\left(x, a \varepsilon^{2}|\log \varepsilon|\right) .
$$

This last inequality together with the fact that $m_{-} \leqslant u_{\varepsilon}$ in $\bar{O} \times(0, T)$ finally gives

$$
\left(m_{+}-\beta \varepsilon\right) \mathbb{1}_{\{d(x, 0) \geqslant \delta\}}+m_{-} \mathbb{1}_{\{d(x, 0)<\delta\}} \leqslant u_{\varepsilon}\left(\cdot, a \varepsilon^{2}|\log \varepsilon|\right) \quad \text { in } \bar{O} .
$$

The conclusion now follows by choosing $\beta<\delta$ for $\tau=a$.

Proof of Lemma 2.3. We follow the proof of the propagation results in [6] and use the same notations as in the proof of Lemma 2.2. We consider the smooth function $\Phi$ given by 33 and we observe that, for $C>0$ large enough and for some $\alpha>0$, one has

$$
\frac{\partial \Phi}{\partial t}(x, t)+F^{*}\left(x, t, D \Phi, D^{2} \Phi\right)<-\alpha \quad \text { in } O \times(0, T) .
$$

On the other hand, we also have

$$
G(x, t, D \Phi(x, t))<-\alpha
$$

on $\partial O$, in a neighborhood of $\{x: \Phi(x, t)=0\}$ and for small $t$.

Using the smoothness of $\Phi$ and the fact that, for small $t, D \Phi(x, t) \neq 0$ if $\Phi(x, t)=0$, we deduce that there exist $\gamma>0$ and $\bar{h}>0$ small enough such that $d$ is smooth in the set $Q_{\gamma, \bar{h}}=$ $\{(x, t):|d(x, t)| \leqslant \gamma, 0 \leqslant t \leqslant \bar{h}\},|D \Phi| \neq 0$ in $Q_{\gamma, \bar{h}}$ and $d$ satisfies

$$
d_{t}+F^{*}\left(x, t, D d, D^{2} d\right)=d_{t}-\Delta d \leqslant-\frac{\alpha}{2|D \Phi|} \quad \text { in } Q_{\gamma, \bar{h}} .
$$

Recalling the properties of $\Phi$ on $\partial O$, we also have

$$
G(x, t, D d) \leqslant-\frac{\alpha}{2|D \Phi|} \quad \text { on }(\partial O \times[0, \bar{h}]) \cap Q_{\gamma, \bar{h}}
$$

We notice that also

$$
|D d|=1 \quad \text { and } \quad D^{2} d D d=0 \quad \text { in } Q_{\gamma, \bar{h}} .
$$

Next we consider a function of the form

$$
v^{\varepsilon}(x, t)=q\left(\varepsilon^{-1}(d(x, t)-2 \beta)\right)-2 \beta \varepsilon,
$$

where $q$ is the traveling wave given by [25]. By analogous computations to the ones of [6], one can see that if $\beta$ is small enough then $v^{\varepsilon}$ satisfies, for some constant $v(\alpha, \beta)<0$,

$$
v_{t}^{\varepsilon}-\Delta v^{\varepsilon}+\varepsilon^{-2} f\left(v^{\varepsilon}\right) \leqslant \varepsilon^{-1} v(\alpha, \beta)+O(1) \quad \text { as } \varepsilon \rightarrow 0,
$$

for all $(x, t) \in\{|d(x, t)| \leqslant \gamma, 0 \leqslant t \leqslant \bar{h}\}$.

Moreover we observe that, by construction, for all $(x, t) \in(\partial O \times[0, T]) \cap Q_{\gamma, \bar{h}}$ we have $D v^{\varepsilon}(x, t)=(\dot{q} / \varepsilon) D d(x, t)$ and thus $G\left(x, t, D v^{\varepsilon}(x, t)\right)=(\dot{q} / \varepsilon) G(x, t, D d(x, t)) \leqslant 0$ because of (A4) and 41. 
Now we have to extend the subsolution $v^{\varepsilon}$ to $\bar{O} \times[0, \bar{h}]$. We do this in two steps. The first step is to define the function $\bar{v}^{\varepsilon}:\{(x, t) \in \bar{O} \times[0, \bar{h}]: d(x, t) \leqslant \gamma\} \rightarrow \mathbb{R}$ by

$$
\bar{v}^{\varepsilon}(x, t)= \begin{cases}\sup \left(v^{\varepsilon}(x, t), m_{-}\right) & \text {if } d(x, t)>-\gamma, \\ m_{-} & \text {if } d(x, t) \leqslant-\gamma .\end{cases}
$$

By similar computations to those of Lemma 4.4 in [6] one proves that $\bar{v}^{\varepsilon}$ is a viscosity subsolution of (23).

Then we choose a smooth function $\psi: \mathbb{R} \rightarrow \mathbb{R}$ such that $\psi^{\prime} \leqslant 0$ in $\mathbb{R}, \psi=1$ in $(-\infty, \gamma / 2)$, $0<\psi<1$ in $(\gamma / 2,3 \gamma / 4), \psi=0$ in $(3 \gamma / 4, \infty)$, and finally, $\psi^{\prime \prime} \leqslant 0$ in a neighborhood of $\gamma / 2$. The function $w^{\varepsilon, \beta}: \bar{O} \times[0, \bar{h}] \rightarrow \mathbb{R}$ defined by

$$
w^{\varepsilon, \beta}(x, t)= \begin{cases}\psi(d(x, t)) \bar{v}^{\varepsilon}(x, t)+(1-\psi(d(x, t)))\left(m_{+}-\beta \varepsilon\right) & \text { if } d(x, t)<\gamma, \\ m_{+}-\beta \varepsilon & \text { otherwise, }\end{cases}
$$

is a viscosity subsolution of 23) in $O \times[0, \bar{h}]$ if $\varepsilon$ and $\bar{h}$ are sufficiently small. Moreover,

$$
w^{\varepsilon, \beta}(\cdot, 0) \leqslant\left(m_{+}-\beta \varepsilon\right) \mathbb{1}_{\{d(x, 0) \geqslant \beta\}}+m_{-} \mathbb{1}_{\{d(x, 0)<\beta\}} \quad \text { in } \bar{O} .
$$

We have to check the subsolution property only on the set $\{\gamma / 2<d(x, t)<3 \gamma / 4\}$. In order to simplify the notations, we drop the superscript " $\varepsilon, \beta$ " on $w$ as well as the superscript " $\varepsilon$ " on $\bar{v}$. We have

$$
\begin{aligned}
w_{t}-\Delta w+\varepsilon^{-2} f(w)= & \psi\left(\bar{v}_{t}-\Delta \bar{v}\right)+\left[\psi^{\prime}\left(d_{t}-\Delta d\right)-\psi^{\prime \prime}\right]\left(\bar{v}-\left(m_{+}-\beta \varepsilon\right)\right) \\
& -2 \psi^{\prime} D d \cdot D \bar{v}+\varepsilon^{-2} f\left(\psi \bar{v}+(1-\psi)\left(m_{+}-\beta \varepsilon\right)\right),
\end{aligned}
$$

where we have dropped the arguments of the function $\psi$ for the sake of clarity.

Since we are arguing in the set $\{\gamma / 2<d(x, t)<3 \gamma / 4\}$, using the asymptotic behavior of $q$ at $\infty$, we find that, for some constant $\tilde{c}>0$,

$$
\bar{v}(x, t)=m_{+}-\exp \left(-(\varepsilon)^{-1} \tilde{c}\right)-2 \beta \varepsilon=m_{+}-2 \beta \varepsilon+o(\varepsilon) \quad \text { as } \varepsilon \rightarrow 0
$$

and hence, for $\varepsilon$ small enough,

$$
\bar{v}(x, t)-\left(m_{+}-\beta \varepsilon\right)=-\beta \varepsilon+o(\varepsilon) \leqslant 0 .
$$

Since $\psi^{\prime} \leqslant 0$ in $\mathbb{R}$ and $d_{t}-\Delta d<0$, we also have

$$
\psi^{\prime}\left(d_{t}-\Delta d\right)\left(\bar{v}-\left(m_{+}-\beta \varepsilon\right)\right) \leqslant 0 .
$$

But $f$ is convex in a neighborhood of $m_{+}$. Therefore, if $\varepsilon$ is sufficiently small,

$$
f(w) \leqslant \psi f(\bar{v})+(1-\psi) f\left(m_{+}-\beta \varepsilon\right) .
$$

Substituting all this information in 443 yields

$$
\begin{aligned}
w_{t}-\Delta w+\varepsilon^{-2} f(w) \leqslant & \psi \frac{\nu(\beta, \alpha)}{\varepsilon}-2 \psi^{\prime} D d \cdot D \bar{v} \\
& -\psi^{\prime \prime}\left(\bar{v}-\left(m_{+}-\beta \varepsilon\right)\right)+(1-\psi) \varepsilon^{-2} f\left(m_{+}-\beta \varepsilon\right) .
\end{aligned}
$$


By hypothesis $\psi^{\prime \prime}(s) \leqslant 0$ if $s \leqslant \gamma / 2+\mu$ for some $\mu>0$, thus for $d(x, t) \leqslant \mu+\gamma / 2$, the right-hand side of (45) is negative for $\varepsilon$ small enough since it is of the form $O(1)+\psi v(\beta, \alpha) / \varepsilon$ as $\varepsilon \rightarrow 0$.

If $s>\mu+\gamma / 2$, then $1-\psi(s) \geqslant c(\mu)>0$; hence,

$$
w_{t}-\Delta w+\varepsilon^{-2} f(w) \leqslant O(1)+c(\mu) \varepsilon^{-2} f\left(m_{+}-\beta \varepsilon\right) .
$$

The right-hand side of this last inequality is negative for $\varepsilon$ small enough, since $f\left(m_{+}\right)=0$ and $f^{\prime}\left(m_{+}\right)>0$.

For the Neumann type boundary condition in the set $\{\gamma / 2<d(x, t)<3 \gamma / 4\} \cap \partial O$, we note that, for $\varepsilon$ small enough,

$$
D w(x, t)=\left(\psi^{\prime}\left(\bar{v}-m_{+}+\beta \varepsilon\right)+\psi \dot{q} / \varepsilon\right) D d .
$$

Because of the properties of $\psi$, the fact that $\dot{q} \geqslant 0$ in $\mathbb{R}$ and 44 , the quantity $\psi^{\prime}\left(\bar{v}-m_{+}+\beta \varepsilon\right)+$ $\psi \dot{q} / \varepsilon$ is positive in the set $\{\gamma / 2<d(x, t)<3 \gamma / 4\} \cap \partial O$ and therefore

$$
G(x, t, D w)=\left(\psi^{\prime}\left(\bar{v}-m_{+}+\beta \varepsilon\right)+\psi \dot{q} / \varepsilon\right) G(x, t, D d) \leqslant 0
$$

hence $w$ satisfies the Neumann boundary condition.

Finally, using the form of the function $w=w^{\varepsilon, \beta}$ we have built, it is clear that if $(x, t) \in$ $B \bar{O}\left(x_{0}, r\right) \times(0, \bar{h})$ satisfies $d(x, t)>2 \beta$, then

$$
\lim \inf _{*}\left[\frac{w^{\varepsilon, \beta}-m_{+}}{\varepsilon}\right](x, t) \geqslant-2 \beta .
$$

Thus the proof of Lemma 2.3 is complete.

STEP 2: Propagation. Next we show that $\left(\Omega_{t}^{1}\right)_{t}$ is a generalized superflow:

Proposition 2.2 (Propagation II: local version) Let $x_{0} \in \bar{O}, t \in(0, T), r>0, h>0$ be such that $t+h<T$ and let $\phi: \bar{O} \times[0, T] \rightarrow \mathbb{R}$ be a smooth function such that, for some $\alpha>0$,

(i) $\frac{\partial \phi}{\partial t}+F^{*}\left(y, s, D \phi, D^{2} \phi\right)<-\alpha$ on $\bar{B} \bar{O}\left(x_{0}, r\right) \times[t, t+h]$,

(ii) $G(y, s, D \phi)<-\alpha$ on $\partial O \cap \bar{B}\left(x_{0}, r\right) \times[t, t+h]$,

(iii) for any $t \leqslant s \leqslant t+h,\left\{y \in B \bar{O}\left(x_{0}, r\right): \phi(y, s)=0\right\} \neq \emptyset$ and

$$
|D \phi(y, s)| \neq 0 \quad \text { in }\left\{(y, s) \in \bar{B} \bar{O}\left(x_{0}, r\right) \times[t, t+h]: \phi(y, s)=0\right\},
$$

(iv) $\left\{y \in \bar{B} \bar{O}\left(x_{0}, r\right): \phi(y, t) \geqslant 0\right\} \subset \Omega_{t}$,

(v) for all $s \in[t, t+h],\left\{y \in \partial B \bar{O}\left(x_{0}, r\right): \phi(y, s) \geqslant 0\right\} \subset \Omega_{s}$.

Then, for all $x \in B \bar{O}\left(x_{0}, r\right)$ such that $\phi(x, t+h)>0$, we have

$$
\liminf { }_{*}\left[\frac{u^{\varepsilon}-m_{+}}{\varepsilon}\right](y, s) \geqslant 0
$$

for $(y, s)$ in a small neighborhood of $(x, t+h)$ and therefore $(x, t+h) \in \Omega_{t+h}^{1} \times\{t+h\}$. 
Proof. The argument follows the proof of Lemma 2.3 with a key difference: for $\beta, \varepsilon$ small enough, we are going to build a subsolution $\omega^{\varepsilon, \beta}$ of 23 only in the ball $\bar{B} \bar{O}\left(x_{0}, r\right)$ and not in the whole domain $\bar{O}$, condition (v) providing some kind of Dirichlet boundary condition on $\partial B \bar{O}\left(x_{0}, r\right)$. Because of this similarity, we just sketch the proof.

Our aim is to build a subsolution $\omega^{\varepsilon, \beta}$ of 23 in $\bar{B} \bar{O}\left(x_{0}, r\right) \times[t, t+h]$, satisfying

$$
\omega^{\varepsilon, \beta}(\cdot, t) \leqslant\left(m_{+}-\beta \varepsilon\right) \mathbb{1}_{\{d(\cdot, t) \geqslant \beta\}}+m_{-} \mathbb{1}_{\{d(\cdot, t)<\beta\}} \quad \text { in } \bar{B} \bar{O}\left(x_{0}, r\right),
$$

and for all $s \in[t, t+h]$,

$$
\omega^{\varepsilon, \beta}(\cdot, s) \leqslant\left(m_{+}-\beta \varepsilon\right) \mathbb{1}_{\{d(\cdot, s) \geqslant \beta\}}+m_{-} \mathbb{1}_{\{d(\cdot, s)<\beta\}} \quad \text { on } \partial B \bar{O}\left(x_{0}, r\right),
$$

where, for all $s \in[t, t+h], d(\cdot, s)$ is the signed distance to the set $\{\phi(\cdot, s)=0\}$ which has the same sign as $\phi$. Moreover we require that if $(x, s) \in \bar{B} \bar{O}\left(x_{0}, r\right) \times[t, t+h]$ satisfies $d(x, s)>2 \beta$, then

$$
\liminf _{*}\left[\frac{\omega^{\varepsilon, \beta}-m_{+}}{\varepsilon}\right](x, s) \geqslant-2 \beta .
$$

Because of the hypotheses on $\phi$, there exists $\gamma$ such that $d$ is smooth in the set $Q_{\gamma}=\{(x, s) \in$ $\left.\bar{B}_{\bar{O}}\left(x_{0}, r\right) \times[t, t+h]:|d(x, s)|<\gamma\right\},|D \phi(x, s)| \neq 0$ in $Q_{\gamma}$ and

$$
d_{t}+F^{*}\left(x, s, D d, D^{2} d\right)=d_{t}-\Delta d \leqslant-\frac{\alpha}{4|D \phi|} \quad \text { in } Q_{\gamma},
$$

and

$$
G(x, s, D d) \leqslant-\frac{\alpha}{4|D \phi|} \quad \text { on }(\partial O \times[t, t+h]) \cap Q_{\gamma} .
$$

We consider in $Q_{\gamma}$ the function $v^{\varepsilon}$ of the form (42). By the arguments of Lemmas 3.2 and 4.2 in [6] one shows that $v^{\varepsilon}$ satisfies 23 in $Q_{\gamma}$.

The next point consists in extending the subsolution $v^{\varepsilon}$ to the whole domain $\bar{B} \bar{O}(x, r) \times[t, t+h]$. We do this as in the proof of Lemma 2.3 in a series of lemmas whose proofs are left to the reader.

LEMMA 2.4 For $\varepsilon$ sufficiently small, the function $\bar{v}^{\varepsilon}$ defined on $\left\{(x, s) \in \bar{B} \bar{O}\left(x_{0}, r\right) \times[t, t+h]\right.$ : $d(x, s) \leqslant \gamma\}$ by

$$
\bar{v}^{\varepsilon}(x, s)= \begin{cases}\sup \left(v^{\varepsilon}(x, s), m_{-}\right) & \text {in }\left\{(x, s) \in \bar{B} \bar{O}\left(x_{0}, r\right) \times[t, t+h]: d(x, s)>-\gamma\right\}, \\ m_{-} & \text {in }\left\{(x, s) \in \bar{B} \bar{O}\left(x_{0}, r\right) \times[t, t+h]: d(x, s) \leqslant-\gamma\right\},\end{cases}
$$

is a viscosity subsolution of $[23)$ in $\left\{(x, s) \in \bar{B} \bar{O}\left(x_{0}, r\right) \times[t, t+h]: d(x, s)<\gamma\right\}$.

Next we consider the function $\psi$ defined in the proof of Lemma 2.3

LEMma 2.5 The function $\omega^{\varepsilon, \beta}: \bar{B} \bar{O}\left(x_{0}, r\right) \times[t, t+h] \rightarrow \mathbb{R}$ defined by

$$
\omega^{\varepsilon, \beta}(x, s)= \begin{cases}\psi(d(x, s)) \bar{v}^{\varepsilon}(x, s)+(1-\psi(d(x, s)))\left(m_{+}-\beta \varepsilon\right) & \text { in }\{d(x, s)<\gamma\}, \\ m_{+}-\beta \varepsilon & \text { elsewhere, }\end{cases}
$$

is a viscosity subsolution of $[23)$ in $\bar{B} \bar{O}\left(x_{0}, r\right) \times[t, t+h]$ if $\varepsilon$ is small enough. Moreover,

$$
\omega^{\varepsilon, \beta}(\cdot, t) \leqslant\left(m_{+}-\beta \varepsilon\right) \mathbb{1}_{\{d(\cdot, t) \geqslant \beta\}}+m_{-} \mathbb{1}_{\{d(\cdot, t)<\beta\}} \quad \text { in } \bar{B} \bar{O}\left(x_{0}, r\right),
$$


and for all $s \in[t, t+h]$,

$$
\omega^{\varepsilon, \beta}(\cdot, s) \leqslant\left(m_{+}-\beta \varepsilon\right) \mathbb{1}_{\{d(\cdot, s) \geqslant \beta\}}+m_{-} \mathbb{1}_{\{d(\cdot, s)<\beta\}} \quad \text { on } \partial B \bar{O}\left(x_{0}, r\right) .
$$

Finally, for all $(x, s) \in B_{\bar{O}}\left(x_{0}, r\right) \times[t, t+h]$ such that $d(x, s)>2 \beta$, we have

$$
\liminf { }_{*}\left[\frac{\omega^{\varepsilon, \beta}-m_{+}}{\varepsilon}\right](x, s) \geqslant-2 \beta .
$$

Now we conclude the proof of Proposition 2.2 by using the two lemmas. Consider the subsolution $\omega^{\varepsilon, \beta}$ given by Lemma 2.5 . It remains to check that, for $\varepsilon$ small enough, $u_{\varepsilon}$ satisfies

$$
u_{\varepsilon}(x, t) \geqslant\left(m_{+}-\beta \varepsilon\right) \mathbb{1}_{\{d(x, t) \geqslant \beta\}}+m_{-} \mathbb{1}_{\{d(x, t)<\beta\}} \quad \text { in } \bar{B} \bar{O}\left(x_{0}, r\right),
$$

and for all $s \in[t, t+h]$,

$$
u_{\varepsilon}(x, s) \geqslant\left(m_{+}-\beta \varepsilon\right) \mathbb{1}_{\{d(x, s) \geqslant \beta\}}+m_{-} \mathbb{1}_{\{d(x, s)<\beta\}} \quad \text { on } \partial B_{\bar{O}}\left(x_{0}, r\right) .
$$

The inequalities 48 and 49 follow respectively from the fact that $\left\{y \in \bar{B} \bar{O}\left(x_{0}, r\right): \phi(y, t) \geqslant 0\right\}$ $\subset \Omega_{t}$, and from $\left\{y \in \partial B \bar{O}\left(x_{0}, r\right): \phi(y, s) \geqslant 0\right\} \subset \Omega_{s}$ for all $s \in[t, t+h]$ and the compactness of these two $\phi$-sets.

Thus, by the maximum principle, for $\varepsilon$ small enough depending on $\beta$ and $\phi$, we have

$$
\omega^{\varepsilon, \beta} \leqslant u_{\varepsilon} \quad \text { in } \bar{B} \bar{O}\left(x_{0}, r\right) \times[t, t+h] .
$$

At this point, we remark that if $\phi$ satisfies conditions (i)-(v) of Proposition 2.2 in $\bar{B} \bar{O}\left(x_{0}, r\right) \times$ $[t, t+h]$, it also satisfies them in a slightly larger time interval $\left[t, t+h^{\prime}\right], h^{\prime}>h$. Therefore the subsolution $\omega^{\varepsilon, \beta}$ can be built in $\bar{B} \bar{O}\left(x_{0}, r\right) \times\left[t, t+h^{\prime}\right]$ and the above inequality holds in this larger domain.

We observe that because of the form $\omega^{\varepsilon, \beta}$, for all $x \in B \bar{O}\left(x_{0}, r\right)$ such that $d(x, t+h)>2 \beta$, we have

$$
\liminf _{*}\left[\frac{\omega^{\varepsilon, \beta}-m_{+}}{\varepsilon}\right](x, t+h) \geqslant-2 \beta .
$$

Therefore for all $x \in B \bar{O}\left(x_{0}, r\right)$ such that $d(x, t+h)>2 \beta$, the inequality 50 yields

$$
\liminf _{*}\left[\frac{u_{\varepsilon}-m_{+}}{\varepsilon}\right](x, t+h) \geqslant-2 \beta .
$$

Since $\beta$ is arbitrary, we have

$$
\left\{x \in B \bar{O}\left(x_{0}, r\right): \phi(x, t+h)>0\right\} \subset \Omega_{t+h}^{1},
$$

which is exactly the inclusion we wanted to prove.

\subsection{Extensions to nonlinear diffusions and $(x, t)$-dependent reaction terms}

We start with the case of the nonlinear Allen-Cahn equation of the form

$$
u_{\varepsilon, t}-\operatorname{tr}\left(A\left(x, \widehat{D u}_{\varepsilon}\right) D^{2} u_{\varepsilon}\right)+\varepsilon^{-2} f\left(u_{\varepsilon}\right)=0 \quad \text { in } O \times(0, \infty),
$$


where we recall that $\hat{q}:=q /|q|$ for $q \in \mathbb{R}^{N} \backslash\{0\}$ and where $f$ is an Allen-Cahn type nonlinearity satisfying the same assumptions as in the previous section and $A$ is a function with values in $\mathcal{S}^{N}$ whose properties are listed below. This equation is associated with the following initial-boundary conditions:

$$
\begin{cases}G\left(x, t, D u_{\varepsilon}\right)=0 & \text { on } \partial O \times(0, \infty), \\ u_{\varepsilon}=g & \text { on } \bar{O} \times\{0\}\end{cases}
$$

where $g$ is a continuous function on $\bar{O}$.

Here the matrix $A=\left(a_{i j}\right)_{i j} \in C^{2}\left(\bar{O} \times \mathbb{R}^{N}, \mathcal{S}^{N}\right)$ is such that

$$
\begin{aligned}
& \text { for all } i, j, k \in\{1, \ldots, N\}, a_{i j}, a_{i j, x_{k}}, a_{i j, p_{k}} \text { are continuous on } \bar{O} \times \mathbb{R}^{N}, \\
& \qquad \begin{array}{l}
\text { for each } R>0 \text { there exists } C_{R}>0 \text { such that for all } p \in \mathbb{R}^{N}, \\
A(\cdot, p) \in W^{2, \infty}\left(\bar{O}, \mathcal{S}^{N}\right) \text { and } \sup _{|p| \leqslant R}\|A(\cdot, p)\|_{W^{2, \infty}} \leqslant C_{R},
\end{array}
\end{aligned}
$$

and, finally, there exists $v>0$ such that for all $(x, p, q) \in \bar{O} \times \mathbb{R}^{N} \backslash\{0\} \times \mathbb{R}^{N}$,

$$
A(x, \hat{p}) q \cdot q \geqslant v|q|^{2} .
$$

To state the result about the asymptotics of (51)-(52) we need to recall that, for every $u_{0} \in$ $C(\bar{O})$, the initial value problem

$$
\left\{\begin{array}{l}
u_{t}+F\left(x, D u, D^{2} u\right)=0 \quad \text { in } O \times(0, \infty) \\
G(x, t, D u)=0 \quad \text { on } \partial O(0, \infty) \\
u=u_{0} \quad \text { on } O \times\{0\}
\end{array}\right.
$$

with

$$
\begin{aligned}
F(x, p, X)= & -\operatorname{tr}\left\{A(x, \hat{p}) X\left[I-(A(x, \hat{p}) p \cdot p)^{-1}(A p \otimes p)\right]\right\} \\
& +(2 A(x, \hat{p}) p \cdot p)^{-1} \operatorname{tr}\left\{A ( x , \hat { p } ) p \otimes \left[D_{x} A(x, \hat{p}) p \cdot p\right.\right. \\
& \left.\left.+|p|^{-1}(X-X \hat{p} \otimes \hat{p}) D_{p} A(x, \hat{p}) p \cdot p\right]\right\},
\end{aligned}
$$

has a unique viscosity solution $u \in C(\bar{O} \times[0, T])$ for all $T>0$. The proof of this fact, which is true under suitable assumptions on $G$, can be found in [3].

TheOREM 2.2 Assume (24), 25, (53), 54, (55) and let $u_{\varepsilon}$ be the solution of (51)-(52) with $g: \mathbb{R}^{N} \rightarrow \mathbb{R}$ such that $\Gamma_{0}=\left\{x: g(x)=m_{0}\right\}$ is a nonempty subset of $\mathbb{R}^{N}$. Then, as $\varepsilon \rightarrow 0$,

$$
u_{\varepsilon}(x, t) \rightarrow \begin{cases}m_{+} & \text {locally uniformly in } \\ m_{-} & \{u>0\},\end{cases}
$$

where $u$ is the unique viscosity solution of 56 with $u_{0}=d_{0}$, the signed distance to $\Gamma_{0}$ such that $d_{0}>0$ in $\left\{g>m_{0}\right\}$ and $d_{0}<0$ in $\left\{g<m_{0}\right\}$.

If, in addition, the no-interior condition (13) holds, then, as $\varepsilon \rightarrow 0$,

$$
u_{\varepsilon}(x, t) \rightarrow \begin{cases}m_{+} & \text {locally uniformly in } \\ m_{-} & \{u>0\}, \\ \{u>0\} & \end{cases}
$$


Proof. The proof follows closely the one of Theorem 2.1 except for a minor additional argument that we give below.

To prove the existence of subsolutions $w^{\varepsilon, \beta}$, we consider a smooth function $\phi$ satisfying the conditions of Definition 1.1 and we set, as in Section 1,

$$
v^{\varepsilon}(x, s)=q\left(\varepsilon^{-1}(\tilde{d}(x, s)-2 \beta)\right)-\beta \varepsilon,
$$

where for all $s>0, \tilde{d}(\cdot, s)$ is a different signed-distance function to the set $\{\phi(x, s)=0\}$ since it solves

$$
A(x, \widehat{D \tilde{d}}) D \tilde{d} \cdot D \tilde{d}=1
$$

in $\{(x, s) \in O \times[t, t+h]:|\phi(x, s)| \leqslant \gamma\}$ with $\gamma>0$ small enough. The function $\tilde{d}$ is smooth in this set if $\gamma$ is small enough as a consequence of the method of characteristics.

With this new distance function, all the computations of Section 2.2 extend easily to this more complicated case.

We now consider the more complicated case of $(x, t)$-dependent nonlinearities. More specifically, we study the asymptotic behavior as $\varepsilon \rightarrow 0$ of the solutions of the equations

$$
u_{\varepsilon, t}-\Delta u_{\varepsilon}+b(x) \cdot D u_{\varepsilon}+\varepsilon^{-2} f^{\varepsilon}\left(u_{\varepsilon}, x, t\right)=0 \quad \text { in } O \times(0, \infty),
$$

and

$$
u_{\varepsilon, t}-\varepsilon \Delta u_{\varepsilon}+b(x) \cdot D u_{\varepsilon}+\varepsilon^{-1} f^{\varepsilon}\left(u_{\varepsilon}, x, t\right)=0 \quad \text { in } O \times(0, \infty),
$$

where $b: \bar{O} \rightarrow \mathbb{R}^{N}$ is a Lipschitz continuous vector field and the functions $u \mapsto f^{\varepsilon}(u, x, t)$ are $(x, t)$-dependent "cubic-type" nonlinearities satisfying suitable assumptions. As in the Allen-Cahn case, we consider the equations (58) and (59) together with (52).

The two model cases we have in mind are

$$
f^{\varepsilon}(u, x, t)=f(u)+\varepsilon \theta(x, t)
$$

for (58) with $f$ satisfying (24), and

$$
f^{\varepsilon}(u, x, t)=2(u-\mu(x, t))\left(u-m_{-}\right)\left(u-m_{+}\right)+\varepsilon \theta(x, t)
$$

for 59), where, say, $\theta, \mu \in W^{1, \infty}(\bar{O} \times[0, \infty))$ and $\mu$ takes values in $\left(m_{-}, m_{+}\right)$.

In the general case, we assume that the functions $f^{\varepsilon}$ depend continuously on $\varepsilon \geqslant 0$, are $C^{2}$ functions of $u, x, C^{1}$-functions of $t$ and that for sufficiently small $\varepsilon \geqslant 0$ there exist $h_{-}^{\varepsilon}(x, t)<$ $h_{0}^{\varepsilon}(x, t)<h_{+}^{\varepsilon}(x, t)$ such that

$$
f^{\varepsilon}\left(h_{-}^{\varepsilon}(x, t), x, t\right)=f^{\varepsilon}\left(h_{+}^{\varepsilon}(x, t), x, t\right)=f^{\varepsilon}\left(h_{0}^{\varepsilon}(x, t), x, t\right)=0,
$$

with, for any $x \in \bar{O}$ and $t>0$,

$$
f^{\varepsilon}(s, x, t)>0 \quad \text { on }\left(h_{-}^{\varepsilon}(x, t), h_{0}^{\varepsilon}(x, t)\right), \quad f^{\varepsilon}(s, x, t)<0 \quad \text { on }\left(h_{0}^{\varepsilon}(x, t), h_{+}^{\varepsilon}(x, t)\right),
$$

and

$$
f_{u}^{\varepsilon}(u, x, t) \geqslant \gamma>0 \quad \text { on }\left(-\infty, h_{-}^{\varepsilon}(x, t)+\gamma\right] \cup\left[h_{+}^{\varepsilon}(x, t)-\gamma, \infty\right),
$$

for some $\gamma>0$ independent of $(x, t, \varepsilon)$. 
Depending on whether we consider (58) or (59), we impose two different types of assumptions on the derivatives of the functions $f^{\varepsilon}$, namely, we assume that, either for $\kappa=0$ or 1 , for any compact subset $K$ of $\mathbb{R} \times \bar{O} \times[0, \infty)$, there exists a constant $C(K)>0$ such that, for $\varepsilon$ small enough and for all $(r, x, t) \in K, 1 \leqslant i, j \leqslant N$,

$$
\left|D_{t} f^{\varepsilon}(r, x, t)\right|,\left|D_{x_{i}} f^{\varepsilon}(r, x, t)\right|,\left|D_{x_{i} x_{j}} f^{\varepsilon}(r, x, t)\right|,\left|D_{x_{i}} f^{\varepsilon}(r, x, t)\right| \leqslant C(K) \varepsilon^{\kappa},
$$

and

$$
\left|D_{r} f^{\varepsilon}(r, x, t)\right|,\left|D_{r r} f^{\varepsilon}(r, x, t)\right| \leqslant C(K) .
$$

To simplify the notations and to make them agree with the Allen-Cahn case, we write $m_{ \pm}(x, t):=h_{ \pm}^{0}(x, t)$ and $m_{0}(x, t):=h_{0}^{0}(x, t)$ for $x \in \bar{O}$ and $t \geqslant 0$. As a consequence of the above assumptions on $f^{\varepsilon}$, we have

$$
h_{ \pm}^{\varepsilon}(x, t) \rightarrow m_{ \pm}(x, t), \quad h_{0}^{\varepsilon}(x, t) \rightarrow m_{0}(x, t) \quad \text { as } \varepsilon \rightarrow 0,
$$

uniformly on compact subsets of $\bar{O} \times[0, \infty)$, where, in fact, $m_{ \pm}$and $m_{0}$ do not depend on $x$ and $t$ if $\kappa=1$.

Since, for fixed $(x, t, \varepsilon)$, the function $u \mapsto f^{\varepsilon}(u, t, x)$ satisfies the hypotheses of Aronson and Weinberger [2] and Fife and McLeod [12], there exists a unique pair $\left(q^{\varepsilon}(r, x, t), c^{\varepsilon}(x, t)\right)$ such that

$$
q_{r r}^{\varepsilon}(r, x, t)+c^{\varepsilon}(r, x, t) q_{r}^{\varepsilon}(r, x, t)=f^{\varepsilon}\left(q^{\varepsilon}(r, x, t), x, t\right)
$$

and

$$
\lim _{r \pm \infty} q^{\varepsilon}(r, x, t)=h_{ \pm}^{\varepsilon}(x, t), \quad q^{\varepsilon}(0, x, t)=h_{0}^{\varepsilon}(x, t) .
$$

In the particular case of (60) and (61), we have an explicit formula for $\left(q^{\varepsilon}(r, x, t), c^{\varepsilon}(x, t)\right)$, namely

$$
\begin{aligned}
q^{\varepsilon}(r, x, t) & =h_{-}^{\varepsilon}(x, t)+m^{\varepsilon}(x, t)\left[1+\exp \left(-m^{\varepsilon}(x, t)\left(r+r^{\varepsilon}(x, t)\right)\right)\right]^{-1}, \\
c^{\varepsilon}(x, t) & =2 h_{0}^{\varepsilon}(x, t)-h_{+}^{\varepsilon}(x, t)-h_{-}^{\varepsilon}(x, t),
\end{aligned}
$$

where $m^{\varepsilon}(x, t)=h_{+}^{\varepsilon}(x, t)-h_{-}^{\varepsilon}(x, t)$ and $r^{\varepsilon}(x, t)$ is such that $q^{\varepsilon}(0, x, t)=h_{0}^{\varepsilon}(x, t)$. Depending on whether we are in the case of (60) or (61), we have different behavior in $\varepsilon$ for the derivatives of $h_{+}^{\varepsilon}(x, t), h_{-}^{\varepsilon}(x, t)$ and $h_{0}^{\varepsilon}(x, t)$ which can be obtained through the Implicit Function Theorem, and this implies different properties of the derivatives of $q^{\varepsilon}$ and $c^{\varepsilon}$.

We continue by listing the technical assumptions on $q^{\varepsilon}$ and $c^{\varepsilon}$ that are used in the statement of our results:

$$
q^{\varepsilon} \text { and } c^{\varepsilon} \text { depend smoothly on } x \text { and } t,
$$

and $q^{\varepsilon}$ satisfies, for all $T>0$ and $r \in \mathbb{R}$ and uniformly w.r.t. $\varepsilon$ and $(x, t) \in \bar{O} \times[0, T]$,

(i) $q_{r}^{\varepsilon}(r, x, t)>0$,

(ii) $q^{\varepsilon}(r, x, t) \rightarrow h_{ \pm}^{\varepsilon}(x, t)$ exponentially fast as $r \rightarrow \pm \infty$,

(iii) $q_{t}^{\varepsilon}, \Delta_{x} q^{\varepsilon}=O(1), D_{x} q^{\varepsilon}=O\left(\varepsilon^{\kappa}\right), D_{x} q_{r}^{\varepsilon}=o(1)$ as $\varepsilon \rightarrow 0$.

Finally, if 63 holds with $\kappa=0$, we assume

$$
c^{\varepsilon}(x, t) \rightarrow \alpha(x, t),
$$


while if it is satisfied with $\kappa=1$, we require

$$
-\varepsilon^{-1} c^{\varepsilon}(x, t) \rightarrow \alpha(x, t),
$$

with all these limits locally uniform in $(x, t)$.

To be concise, we denote by (H0) the above set of hypotheses with $\kappa=0$, and by (H1) the one with $\kappa=1$.

Tedious but straightforward computations show that the functions $f^{\varepsilon}$ given by 60 and the associated $q^{\varepsilon}$ and $c^{\varepsilon}$ satisfy (H1), while in the case of (61) they satisfy (H0).

We expect the limiting behavior of $u_{\varepsilon}$ in $O \times[0, T]$ to be governed in the case of $[58$ by

$$
u_{t}-\operatorname{tr}\left[(I-\widehat{D u} \otimes \widehat{D u}) D^{2} u\right]+b(x) \cdot D u-\alpha(x, t)|D u|=0
$$

with $\alpha(x, t)=\lim _{\varepsilon \rightarrow 0} \varepsilon^{-1} c^{\varepsilon}(x, t)$ if (H1) holds, while in the case of 59) and if (H0) is satisfied, this behavior is expected to be governed by

$$
u_{t}+b(x) \cdot D u-\alpha(x, t)|D u|=0
$$

with $\alpha(x, t)=\lim _{\varepsilon \rightarrow 0} c^{\varepsilon}(x, t)$.

Our main results justify these claims. The first one concerns (58).

THEOREM 2.3 Assume (A1)-(A4) and (H1). If $u_{\varepsilon}$ is the solution of (58)-(52), where the continuous function $g: \bar{O} \rightarrow \mathbb{R}$ is such that $\Gamma_{0}=\left\{x: g(x)=m_{0}\right\}$ is a nonempty subset of $\bar{O}$, then, as $\varepsilon \rightarrow 0$,

$$
u_{\varepsilon}(x, t) \rightarrow \begin{cases}m_{+} & \{u>0\}, \\ m_{-} & \text {locally uniformly in } \\ & \{u<0\},\end{cases}
$$

where $u$ is the unique viscosity solution of

$$
\begin{cases}u_{t}-\operatorname{tr}\left[(I-\widehat{D u} \otimes \widehat{D u}) D^{2} u\right]+b(x) \cdot D u-\alpha(x, t)|D u|=0 & \text { in } O \times(0, \infty), \\ G(x, t, D u)=0 & \text { on } \partial \times(0, \infty), \\ u(x, 0)=d_{0}(x) & \text { on } \bar{O} \times\{0\},\end{cases}
$$

where $d_{0}$ is the signed distance to $\Gamma_{0}$ which is positive in $\left\{g>m_{0}\right\}$ and negative in $\left\{g<m_{0}\right\}$. If, in addition, the no-interior condition (13) holds, then, as $\varepsilon \rightarrow 0$,

$$
u_{\varepsilon}(x, t) \rightarrow\left\{\begin{array}{lll}
m_{+} & \text {locally uniformly in } & \\
m_{-} & \overline{\{u>0\}}^{c} .
\end{array}\right.
$$

For (59) we have

TheOREM 2.4 Assume (A1)-(A4) and (H0). Let $u_{\varepsilon}$ be the solution of 59)-(52), where the continuous function $g: \bar{O} \rightarrow \mathbb{R}$ is such that $\Gamma_{0}=\left\{x: g(x)=m_{0}(x, 0)\right\}$ is a nonempty subset of $\bar{O}$. Then, as $\varepsilon \rightarrow 0$,

$$
u_{\varepsilon}(x, t) \rightarrow \begin{cases}m_{+} & \{u>0\}, \\ m_{-} & \text {locally uniformly in } \\ & \{u<0\},\end{cases}
$$


where $u$ is the unique viscosity solution of

$$
\begin{cases}u_{t}+b(x) \cdot D u-\alpha(x, t)|D u|=0 & \text { in } O \times(0, \infty), \\ G(x, t, D u)=0 & \text { on } \partial \times(0, \infty), \\ u(x, 0)=d_{0}(x) & \text { on } \bar{O} \times\{0\},\end{cases}
$$

where $d_{0}$ is the signed distance to $\Gamma_{0}$ which is positive in $\{g>0\}$ and negative in $\{g<0\}$. If, in addition, the no-interior condition (13) holds, then, as $\varepsilon \rightarrow 0$,

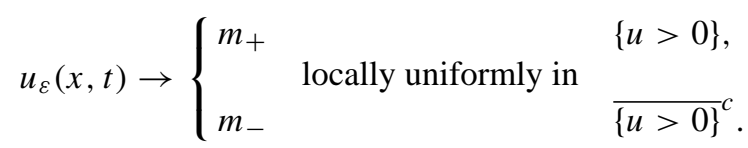

The proofs of Theorems 2.3 and 2.4 follow exactly the same steps as in the proof of Theorem 2.1. we just point out the main changes which are necessary to prove the corresponding step 1 (initialization and global propagation) and step 2 (local propagation),

Proof of Theorem 2.3. We consider the families $\left(\Omega_{t}^{1}\right)_{t}$ and $\left(\Omega_{t}^{2}\right)_{t}$ of sets defined in Section 2.1 by 18, , 19, with $b_{\varepsilon} \equiv h_{+}^{\varepsilon}, a_{\varepsilon} \equiv h_{-}^{\varepsilon}, b=m_{+}, a=m_{-}$and $\tau=1$, and $\Omega_{0}^{1}, \Omega_{0}^{2}$ defined by 22).

In order to describe the main changes to the proof of Theorem 2.1 and to be as concise as possible, we follow exactly the same steps and we use the same notations.

STEP 1: Initialization. We start with the proof of Proposition 2.1 whose statement remains unchanged; to do that, we have to prove the analogues of Lemma 2.2 and 2.3 with $m_{+}$replaced by $h_{+}^{\varepsilon}$ and $m_{-}$by $h_{-}^{\varepsilon}-\beta \varepsilon$.

We first consider the very small time initialization.

1. As in the proof of Lemma 2.2. we have to modify the function $f^{\varepsilon}$, but now taking into account the $(x, t)$-dependence. We proceed in the following way: because of the assumptions on $f^{\varepsilon}$, there exists a function $r \mapsto f_{\delta}(r)$ such that, for every $T>0$, if $\varepsilon$ is small enough, $f_{\delta}(r) \geqslant f^{\varepsilon}(r, x, t)+2 \varepsilon$ for any $r \in \mathbb{R}, x \in \bar{O}$ and $t \in[0, T]$. Moreover $f_{\delta}$ is a cubic-type nonlinearity satisfying 24 with three zeros $m_{-}-\delta, m_{0}+\delta / 2$ and $m_{+}-\delta$.

We modify $f^{\varepsilon}$ in two steps, We first introduce a smooth cut-off function $\zeta_{1} \in C_{0}^{\infty}(\mathbb{R})$ such that $0 \leqslant \zeta_{1} \leqslant 1$ in $\mathbb{R}, \zeta_{1}(r)=1$ in $\left(m_{0}-\delta, m_{0}+\delta\right)$ and $\zeta_{1}(r)=0$ for $r \leqslant m_{0}-2 \delta$ and $r \geqslant m_{0}+2 \delta$. We set,

$$
\tilde{f}_{\delta}^{\varepsilon}(r, x, t)=\zeta_{1}(r) f_{\delta}(r)+\left(1-\zeta_{1}(r)\right)\left[f^{\varepsilon}(r, x, t)+\varepsilon \beta \varphi\left(-C \beta^{-1} \bar{\delta}(x)\right)\right],
$$

where $\bar{\delta}(\cdot)$ denotes the distance function to $\partial O, \varphi$ is a $C^{2}$-function which is constant outside a (small) neighborhood of 0 and such that $1 \leqslant \varphi \leqslant 2, \varphi^{\prime}(0)=1$; finally $\beta, C$ are positive constants which will be chosen later on, with at least $\beta \leqslant 1$.

Using the assumptions on $f^{\varepsilon}$, it is easy to see that, for $\delta$ small enough, $\tilde{f}_{\delta}^{\varepsilon}$ has the same regularity properties as $f^{\varepsilon}$ and has exactly three zeros, $h_{-}^{\varepsilon}+O(\beta \varepsilon), m_{0}+\delta / 2, h_{+}^{\varepsilon}+O(\beta \varepsilon)$; moreover $\tilde{f}_{\delta}^{\varepsilon} \geqslant f^{\varepsilon}$ on $\mathbb{R}$ with $\tilde{f}_{\delta}^{\varepsilon}(r)=f^{\varepsilon}(r, x, t)+\varepsilon \beta \varphi\left(-C \beta^{-1} \bar{\delta}(x)\right)$ if $\left|r-m_{0}\right| \geqslant 2 \delta$. Two key properties of $\tilde{f}_{\delta}^{\varepsilon}$ are: $\tilde{f}_{\delta}^{\varepsilon}$ is independent of $x$ and $t$ for $\left|r-m_{0}\right| \leqslant \delta$, and if we choose $C>0$ large enough, we have, for all $r \in \mathbb{R}, x \in \partial O$ and $t \in[0, T]$,

$$
D_{x} \tilde{f}_{\delta}^{\varepsilon}(r, x, t) \cdot n(x) \geqslant K(T)\left|\mathcal{T}\left(D_{x} \tilde{f}_{\delta}^{\varepsilon}(r, x, t)\right)\right|,
$$


where $K(T)$ is the constant given by Lemma 2.1. Indeed, (77) is a consequence of the form of the $\varphi$-term and of 63) which yields $D_{x} f^{\varepsilon}=O(\varepsilon)$.

2. Then we consider another cut-off function $\zeta_{2} \in C_{0}^{\infty}(\mathbb{R})$ such that $0 \leqslant \zeta_{2} \leqslant 1$ in $\mathbb{R}, \zeta_{2}(s)=0$ in $\left(-\infty, m_{0}+\delta / 4\right] \cup\left[m_{0}+\delta, \infty\right)$ and $\zeta_{2}(s)=1$ in $\left[m_{0}+\delta / 3, m_{0}+2 \delta / 3\right]$. Finally we consider

$$
\bar{f}_{\delta}^{\varepsilon}(r, x, t)=\left(1-\zeta_{2}(s)\right) \tilde{f}_{\delta}^{\varepsilon}(r, x, t)+\zeta_{2}(r) \frac{\delta / 2+m_{0}-s}{|\log \varepsilon|} .
$$

We note that, because again of the properties of $f^{\varepsilon}, \bar{f}_{\delta}^{\varepsilon}$ has exactly three zeros: $h_{+}^{\varepsilon}+O(\beta \varepsilon)$, $m_{0}+\delta / 2, h_{+}^{\varepsilon}+O(\beta \varepsilon)$; moreover, for $\varepsilon$ small enough, $\bar{f}_{\delta}^{\varepsilon} \geqslant f^{\varepsilon}+\beta \varepsilon$ in $\mathbb{R}$ and $\bar{f}_{\delta}^{\varepsilon}=f^{\varepsilon}+\varepsilon \beta \varphi$ for $\left|r-m_{0}\right| \geqslant 2 \delta$. Since this modification of $\tilde{f}_{\delta}^{\varepsilon}$ concerns only a neighborhood of $m_{0}$ (and because of the form of this modification), its key properties are preserved; in particular $\bar{f}_{\delta}^{\varepsilon}$ is independent of $x$ and $t$ for $\left|r-m_{0}\right| \leqslant \delta$ and satisfies a condition analogous to (77).

3. We consider the solution $\chi(\xi, \cdot, x, t)$ of the ode

$$
\left\{\begin{array}{l}
\dot{\chi}+\bar{f}_{\delta}^{\varepsilon}(\chi, x, t)=0 \\
\chi(\xi, 0, x, t)=\xi \in \mathbb{R} .
\end{array}\right.
$$

4. Tedious computations show that the first key properties of $\chi$ remain true, namely

$$
\begin{gathered}
\qquad \chi_{\xi}(\xi, s, x, t)>0 \quad \text { in } \mathbb{R} \times[0, \infty) \times \bar{O} \times[0, \infty), \\
\left\{\begin{array}{l}
\text { for all } \beta>0, T>0, \text { there exists } a(\beta, \delta, T)>0 \text { such that } \\
\chi(\xi, s, x, t) \geqslant h_{+}^{\varepsilon}(x, t)-\beta \varepsilon \quad \text { for } s \geqslant a|\log \varepsilon| \text { and } \xi \geqslant \delta+m_{0} \\
\text { for all }(x, t) \in \bar{O} \times[0, T],
\end{array}\right. \\
\left\{\begin{array}{l}
\text { for every } a, T>0, \text { there exists } M(a, T) \in \mathbb{R} \text { such that, for } \varepsilon \text { small enough, } \\
(\chi \xi(\xi, s, x, t))^{-1}|\chi \xi \xi(\xi, s, x, t)| \leqslant \varepsilon^{-1} M(a, T) \text { for } 0<s \leqslant a|\log \varepsilon|, \\
\text { for all }(x, t) \in \bar{O} \times[0, T] .
\end{array}\right.
\end{gathered}
$$

5. A new point here concerns the behavior of $\chi$ in $x$ and $t$ :

LEMMA 2.6 If $\delta$ is small enough, then for every $a>0$ and $T>0$, there exists $\tilde{M}(a, T)>0$ such that, for $\varepsilon$ small enough and for $0<s \leqslant a|\log \varepsilon|$, we have

$$
\left|\chi_{t}(\xi, s, x, t)\right|,\left|\chi_{x_{i}}(\xi, s, x, t)\right|,\left|\chi_{x_{i} x_{j}}(\xi, s, x, t)\right|,\left|\chi_{\xi x_{i}}(\xi, s, x, t)\right| \leqslant \tilde{M}(a, T) \varepsilon
$$

for any $1 \leqslant i, j \leqslant N$. Moreover, for any $T>0$, if $C>0$ is large enough, then for any $x \in \partial O$, $s \geqslant 0, \xi \in \mathbb{R}$ and $0 \leqslant t \leqslant T$ we have

$$
D_{x} \chi(\xi, s, x, t) \cdot n(x) \leqslant-K(T)\left|\mathcal{T}\left(D_{x} \chi(\xi, s, x, t)\right)\right| .
$$

Proof of Lemma 2.6. This proof uses in an essential way the modifications of $f^{\varepsilon}$ made above. We only prove the estimate for $D_{x} \chi$, the other estimates being proved in the same way.

We consider the ode satisfied by $w:=D_{x} \chi$ obtained by differentiating (78) with respect to $x$, namely

$$
\dot{w}=-D_{r} \bar{f}_{\delta}^{\varepsilon}(\chi, x, t) w-D_{x} \bar{f}_{\delta}^{\varepsilon}(\chi, x, t) .
$$


By assumption $D_{x} \bar{f}_{\delta}^{\varepsilon}(\chi, x, t)=O(\varepsilon)$ as $\varepsilon \rightarrow 0$ but a priori $D_{r} \bar{f}_{\delta}^{\varepsilon}$ is not always positive and this is a difficulty in getting the right estimate.

To overcome it, we use two ingredients: first since $\bar{f}_{\delta}^{\varepsilon}$ depends on $x$ and $t$ only for $\left|r-m_{0}\right| \geqslant \delta$, it is enough to consider initial data $\xi$ for $(78)$ such that $\xi \geqslant m_{0}+\delta$ or $\xi \leqslant m_{0}-\delta$. We only consider the first case, the second one being treated analogously.

If $\delta$ is small enough and $\varepsilon \ll \delta$, then for any $x \in \bar{O}$ and $t \in[0, T], \bar{f}_{\delta}^{\varepsilon} \leqslant-c_{\delta}<0$ for $r \in\left[m_{0}+\delta, m_{+}-\delta\right]$ and $D_{r} \bar{f}_{\delta}^{\varepsilon} \geqslant \gamma / 2>0$ for $r \geqslant m_{+}-\delta$. Therefore, there exists $s_{\delta}>0$, independent of $x$ and $t$, such that if $\xi \geqslant m_{0}+\delta$ and $s \geqslant s_{\delta}$, then $\chi(\xi, s, x, t) \geqslant m_{+}-\delta$. Using this information in 81 , and in particular the fact that $D_{r} \bar{f}_{\delta}^{\varepsilon}(\chi(\xi, s, x, t), x, t) \geqslant \gamma / 2>0$, one easily obtains the desired estimate.

The boundary property for $D_{x} \chi$, though important, is a rather straightforward consequence of 777): indeed, for any vector $e$ such that $e \cdot n(x)=0$ and $|e|=1$, we introduce the function $w:=D_{x} \chi \cdot n(x)+K(T) D_{x} \chi \cdot e$. By looking at the ode satisfied by $w$, namely 81 with $D_{x} \bar{f}_{\delta}^{\varepsilon}$ replaced by $D_{x} \bar{f}_{\delta}^{\varepsilon} \cdot n(x)+K(T) D_{x} \bar{f}_{\delta}^{\varepsilon} \cdot e$, and using 77 , easy arguments show that $w \leqslant 0$ for any $r \geqslant 0, \xi \in \mathbb{R}, x \in \partial O$ and $0 \leqslant t \leqslant T$. Since this is true for any $e$, the result follows.

6. The next step consists in introducing the function $v^{\varepsilon}: \bar{O} \times\left(0, a \varepsilon^{2}|\log \varepsilon|\right) \rightarrow \mathbb{R}$ defined by

$$
v^{\varepsilon}(x, t)=\chi\left(\psi(d(x, 0))-K t / \varepsilon, t / \varepsilon^{2}, x, t\right),
$$

where $d(x, 0)$ is as in Lemma 2.2 and $\psi$ is the function defined by (38) with $m_{-}$replaced by $-\|g\|_{\infty}$ and $m_{0}$ kept unchanged. We are going to verify that $v^{\varepsilon}$ is a viscosity subsolution of (58)-(52).

7. As far as the Neumann boundary condition is concerned, we observe that

$$
D v^{\varepsilon}(x, t)=\chi \xi \psi^{\prime} D d+D_{x} \chi .
$$

By Lemma 2.6 we have $D_{x} \chi \cdot n(x) \leqslant-K(T)\left|\mathcal{T}\left(D_{x} \chi\right)\right|$, thus by applying Lemma 2.1 with $\tilde{p}=$ $\chi_{\xi} \psi^{\prime} D d$ and $p=D_{x} \chi$, it is immediate that the boundary condition is satisfied.

8. Now we check that $v^{\varepsilon}$ satisfies the equation 58 in $\bar{O} \times\left(0, a \varepsilon^{2}|\log \varepsilon|\right)$. We have

$$
\begin{aligned}
& v_{t}^{\varepsilon}-\Delta v^{\varepsilon}+b(x) \cdot D v^{\varepsilon}+\varepsilon^{-2} f^{\varepsilon}(v, x, t)=\chi_{t}+\varepsilon^{-2} \dot{\chi}+b(x) \cdot D_{x} \chi-\Delta_{x} \chi-2 D_{x} \chi \xi \\
& -\chi_{\xi}\left[\varepsilon^{-1} K+\psi^{\prime}+\psi^{\prime \prime} \Delta d+\psi^{\prime 2}(\chi \xi)^{-1} \chi \xi \xi\right. \\
& \left.+b(x) \cdot \psi^{\prime} D d\right]+\varepsilon^{-2}\left(f^{\varepsilon}-\bar{f}_{\delta}^{\varepsilon}\right)+\varepsilon^{-2} \bar{f}_{\delta}^{\varepsilon} \text {. }
\end{aligned}
$$

Given (80) and the fact that, by definition, the function $\psi$ has compact support it is clear that for $K$ large enough the quantity $\varepsilon^{-1} K+\psi^{\prime}+\psi^{\prime \prime} \Delta d+\psi^{\prime 2}\left(\chi_{\xi}\right)^{-1} \chi \xi \xi$ is positive.

As $\dot{\chi}+\bar{f}_{\delta}^{\varepsilon}=0$, it remains to analyze the sign of $\chi_{t}-\Delta_{x} \chi-2 D_{x} \chi_{\xi}+b(x) \cdot D_{x} \chi+\varepsilon^{-2}\left(f^{\varepsilon}-\bar{f}_{\delta}^{\varepsilon}\right)$. To this end we observe that $f^{\varepsilon}-\bar{f}_{\delta}^{\varepsilon} \leqslant-\varepsilon \beta \varphi$. Thus since, by Lemma 2.6, $\chi_{t}, \Delta_{x} \chi, 2 D_{x} \chi_{\xi}$ are $O(\varepsilon)$ as $\varepsilon \rightarrow 0$ locally uniformly in $(x, t) \in \bar{O} \times[0, T]$, we have, for $\varepsilon$ small enough,

$$
\chi_{t}-\Delta_{x} \chi-2 D_{x} \chi \xi+b(x) \cdot D_{x} \chi+\varepsilon^{-2}\left(f^{\varepsilon}-\bar{f}_{\delta}^{\varepsilon}\right) \leqslant 0
$$

and so (58) holds. 
9. We have by construction, on the one hand,

$$
u_{\varepsilon}(x, 0) \geqslant\left(m_{0}+2 \delta\right) \mathbb{1}_{\{d(x, 0) \geqslant \delta\}}-\|g\|_{\infty} \mathbb{1}_{\{d(x, 0)<\delta\}} \quad \text { on } \bar{O},
$$

and, on the other hand,

$$
\begin{aligned}
v^{\varepsilon}(x, 0) & =\chi(\psi(d(x, 0)), 0,0, x)=\psi(d(x, 0)) \\
& \leqslant\left(m_{0}+2 \delta\right) \mathbb{1}_{\{d(x, 0) \geqslant \delta\}}-\|g\|_{\infty} \mathbb{1}_{\{d(x, 0)<\delta\}} .
\end{aligned}
$$

Thus, the maximum principle implies that

$$
v^{\varepsilon}(x, t) \leqslant u_{\varepsilon}(x, t) \quad \text { on } \bar{O} \times\left[0, a \varepsilon^{2}|\log \varepsilon|\right] .
$$

Evaluating $(82)$ for $t_{\varepsilon}=a \varepsilon^{2}|\log \varepsilon|$ and for $x$ such that $d(x, 0) \geqslant \delta$ we get

$$
\chi\left(m_{0}+2 \delta-K a \varepsilon|\log n \varepsilon|, a|\log \varepsilon|, x, a \varepsilon^{2}|\log \varepsilon|\right) \leqslant u_{\varepsilon}\left(x, t_{\varepsilon}\right) .
$$

But, since for $\varepsilon$ small enough,

$$
m_{0}+2 \delta-K a \varepsilon|\log \varepsilon| \geqslant m_{0}+\delta,
$$

it follows from 79 , that

$$
h_{+}^{\varepsilon}\left(x, t_{\varepsilon}\right)+O(\beta \varepsilon) \leqslant u_{\varepsilon}\left(x, t_{\varepsilon}\right) \quad \text { if } d(x, 0) \geqslant \delta .
$$

10. Finally, because of the properties of $\bar{f}_{\delta}^{\varepsilon}$, we also have $\chi\left(\xi, a|\log \varepsilon|, x, a \varepsilon^{2}|\log \varepsilon|\right) \geqslant h_{-}^{\varepsilon}\left(x, t_{\varepsilon}\right)+$ $O(\beta \varepsilon)$ for any bounded $\xi$ if $a$ is large and therefore

$$
h_{-}^{\varepsilon}\left(x, t_{\varepsilon}\right)+O(\beta \varepsilon) \leqslant u_{\varepsilon}\left(x, t_{\varepsilon}\right) \quad \text { for any } x \in \bar{O} .
$$

This gives

$$
\left[h_{+}^{\varepsilon}\left(x, t_{\varepsilon}\right)+O(\beta \varepsilon)\right] \mathbb{1}_{\left\{d\left(x, t_{\varepsilon}\right) \geqslant \delta\right\}}+\left[h_{-}^{\varepsilon}\left(x, t_{\varepsilon}\right)+O(\beta \varepsilon)\right] \mathbb{1}_{\left\{d\left(x, t_{\varepsilon}\right)<\delta\right\}} \leqslant u_{\varepsilon}\left(x, a \varepsilon^{2}|\log \varepsilon|\right) \text { in } \bar{O} .
$$

The conclusion follows by first choosing a smaller $\beta$ if necessary to replace $O(\beta \varepsilon)$ by $\beta \varepsilon$ and then by taking $\delta=\beta$; the result holds for $\tau=a$.

Now we turn to the propagation. The local and global propagation are proved in a similar way, we only consider the global case.

If $t_{\varepsilon}$ is as above, we construct, for $t \geqslant t_{\varepsilon}$, a subsolution $w$ of 58)-(52) such that

$$
w\left(x, t_{\varepsilon}\right) \leqslant\left[h_{+}^{\varepsilon}\left(x, t_{\varepsilon}\right)-\beta \varepsilon\right] \mathbb{1}_{\left\{d\left(x, t_{\varepsilon}\right) \geqslant \beta\right\}}+\left[h_{-}^{\varepsilon}\left(x, t_{\varepsilon}\right)-\beta \varepsilon\right] \mathbb{1}_{\left\{d\left(x, t_{\varepsilon}\right)<\beta\right\}} \quad \text { in } O .
$$

To do that, we follow the argument of the proof of Lemma 2.3 We first consider a function of the form

$$
v^{\varepsilon}(x, t)=q^{\varepsilon}\left(\varepsilon^{-1}(d(x, t)-2 \beta), x, t\right)-2 \beta \varepsilon \varphi\left(-C \beta^{-1} \bar{\delta}(x)\right),
$$

where $q^{\varepsilon}$ is the solution of 66 and, as above, $\varphi$ is a smooth function such that $1 \leqslant \varphi \leqslant 2$, $\varphi^{\prime}(0)=1$. We verify that $v^{\varepsilon}$ is a viscosity solution of $58-25$ in $Q_{\gamma, \bar{h}}$. As far as the boundary condition 52 is concerned, we have

$$
D v^{\varepsilon}(x, t)=\varepsilon^{-1} q_{r}^{\varepsilon} D d+D_{x} q^{\varepsilon}+\varepsilon C \varphi^{\prime} D \bar{\delta}(x) .
$$


We recall that by hypotheses $D_{x} q^{\varepsilon}=O(\varepsilon)$ as $\varepsilon \rightarrow 0$ and that $D \bar{\delta}(x)=-n(x)$, thus for $C$ large enough $D_{x} q^{\varepsilon} \cdot n(x)+\varepsilon C \varphi^{\prime} D \bar{\delta} \cdot n(x) \leqslant-K(T)\left|\mathcal{T}\left(D_{x} q^{\varepsilon} \cdot n(x)+\varepsilon C \varphi^{\prime} D \bar{\delta}\right)\right|$ and so, by using Lemma 2.1. the boundary condition $(52)$ is verified.

Moreover we have

$$
v_{t}^{\varepsilon}-\Delta v^{\varepsilon}+b(x) \cdot D v^{\varepsilon}+\varepsilon^{-2} f^{\varepsilon}\left(v^{\varepsilon}, x, t\right)=\varepsilon^{-2} \mathrm{I}_{\varepsilon}+\varepsilon^{-1} \mathrm{II}_{\varepsilon}+\mathrm{III}_{\varepsilon},
$$

where

$$
\begin{aligned}
\mathrm{I}_{\varepsilon} & =q_{r r}^{\varepsilon}+c^{\varepsilon}(x, t) q_{r}^{\varepsilon}-f^{\varepsilon}\left(q^{\varepsilon}, x, t\right), \\
\mathrm{II}_{\varepsilon} & =q_{r}^{\varepsilon}\left(d_{t}-\Delta d+b(x) \cdot D d+\varepsilon^{-1} c^{\varepsilon}(x, t)\right)-2 D_{x} q_{r}^{\varepsilon} \cdot D d-2 \beta f_{u}^{\varepsilon}\left(q^{\varepsilon}, x, t\right) \varphi, \\
\mathrm{III}_{\varepsilon} & =2 \varepsilon \beta^{-1} C^{2} \varphi^{\prime \prime}-2 \varepsilon C \varphi^{\prime} \Delta \bar{\delta}+b(x) \cdot D_{x} q^{\varepsilon}+q_{t}^{\varepsilon}-\Delta_{x} q^{\varepsilon}+O(1) .
\end{aligned}
$$

We observe that $\mathrm{I}_{\varepsilon}=0$ and by the properties of the traveling wave we have $\mathrm{III}_{\varepsilon}=O(1)$. Thus, by using the properties of the traveling wave and $f_{u}^{\varepsilon}\left(h_{ \pm}^{\varepsilon}, x, t\right) \geqslant \gamma>0$ and the same arguments as in the proof of Lemma 2.3 , one can see that if $\beta$ is small enough then $v^{\varepsilon}$ satisfies, for some constant $v(\alpha, \beta)<0$ (independent of $(x, t)$ ),

$$
v_{t}^{\varepsilon}-\Delta v^{\varepsilon}+\varepsilon^{-2} f^{\varepsilon}\left(v^{\varepsilon}, x, t\right) \leqslant \varepsilon^{-1} v(\alpha, \beta)+O(1) \quad \text { as } \varepsilon \rightarrow 0 .
$$

Next we want to extend the subsolution $v^{\varepsilon}$ to $\bar{O} \times[0, \bar{h}]$ and we do it in two steps.

First we have

LEMmA 2.7 If $C>0$ is a large enough constant, then for $\varepsilon$ small enough, the functions $g_{ \pm}^{\varepsilon}$ defined on $\bar{O} \times[0, \infty)$ by $g_{ \pm}^{\varepsilon}(x, t)=h_{ \pm}^{\varepsilon}(x, t)-\varepsilon \beta \varphi\left(-C \beta^{-1} \bar{\delta}(x)\right)$ are viscosity subsolutions of 558- -52 .

We leave the proof of this claim to the reader since it follows rather easily from the properties of $f^{\varepsilon}, h_{+}^{\varepsilon}$ and $h_{-}^{\varepsilon}$ and from the arguments we used above to prove that $v^{\varepsilon}$ is a subsolution of the equation. We just point out that for the boundary condition $(52)$ we have

$$
D_{x} g_{ \pm}^{\varepsilon}=D_{x} h_{ \pm}^{\varepsilon}(x, t)+C \varepsilon \varphi^{\prime}(0) D \bar{\delta} .
$$

Since, by the properties of $f^{\varepsilon}$ (cf. (63)), $D_{x} h_{ \pm}^{\varepsilon}(x, t)=O(\varepsilon)$ as $\varepsilon \rightarrow 0$, we have $D_{x} g_{ \pm}^{\varepsilon} \cdot n(x)<$ $-K(T)\left|\mathcal{T}\left(D_{x} g_{ \pm}^{\varepsilon}\right)\right|$ for $C$ large enough and thus the boundary condition is satisfied by applying Lemma 2.1 with $\tilde{p}=0$ and $p=D_{x} g_{ \pm}^{\varepsilon}$.

The next step is to define the function $\bar{v}^{\varepsilon}:\left\{(x, t) \in O \times\left[t_{\varepsilon}, t_{\varepsilon}+h\right]: d(x, t) \leqslant \gamma\right\} \rightarrow \mathbb{R}$ by

$$
\bar{v}^{\varepsilon}(x, t)= \begin{cases}\sup \left(v^{\varepsilon}(x, t), g_{-}^{\varepsilon}(x, t)\right) & \text { if } d(x, t)>-\gamma, \\ g_{-}^{\varepsilon}(x, t) & \text { otherwise. }\end{cases}
$$

By similar computations to those of Lemma 4.2 in [6] and using Lemma 2.7] it is easy to prove that $\bar{v}^{\varepsilon}$ is a viscosity subsolution of (58)- $(52)$.

Then we choose a smooth function $\psi: \mathbb{R} \rightarrow \mathbb{R}$ such that $\psi^{\prime} \leqslant 0$ in $\mathbb{R}, \psi=1$ in $(-\infty, \gamma / 2)$, $0<\psi<1$ in $(\gamma / 2,3 \gamma / 4), \psi=0$ in $(3 \gamma / 4, \infty)$, and finally, $\psi^{\prime \prime} \leqslant 0$ in a neighborhood of $\gamma / 2$. The function $w^{\varepsilon}: \bar{O} \times\left[t_{\varepsilon}, t_{\varepsilon}+h\right] \rightarrow \mathbb{R}$ defined by

$$
w^{\varepsilon}(x, t)= \begin{cases}\psi(d(x, t)) \bar{v}^{\varepsilon}(x, t)+(1-\psi(d(x, t))) g_{+}^{\varepsilon}(x, t) & \text { if } d(x, t)<\gamma, \\ g_{+}^{\varepsilon}(x, t) & \text { otherwise }\end{cases}
$$


is a viscosity subsolution of $58-52$ on $\bar{O} \times\left[t_{\varepsilon}, t_{\varepsilon}+h\right]$ if $\varepsilon$ and $h$ are sufficiently small. Moreover

$$
w^{\varepsilon}\left(\cdot, t_{\varepsilon}\right) \leqslant\left(h_{+}^{\varepsilon}\left(\cdot, t_{\varepsilon}\right)-\beta \varepsilon\right) \mathbb{1}_{\left\{d\left(x, t_{\varepsilon}\right) \geqslant \beta\right\}}+\left(h_{-}^{\varepsilon}\left(\cdot, t_{\varepsilon}\right)-\beta \varepsilon\right) \mathbb{1}_{\left\{d\left(x, t_{\varepsilon}\right)<\beta\right\}} \quad \text { in } \bar{O} .
$$

Now the conclusion follows from the maximum principle, which allows us to compare $u^{\varepsilon}$ and $w^{\varepsilon}$, and from the form of the function $w^{\varepsilon}$.

Proof of Theorem 2.4. We only give a very brief sketch of the proof since it is based on the same arguments as the proof of Theorem 2.3 (or even simpler). The main change (and this will simplify matters) is that, roughly speaking, the term $\beta \varepsilon$ is replaced everywhere by $\beta$.

The main change in the proof (which explains why we work with $\beta$ instead of $\beta \varepsilon$ ) is that we now consider the families $\left(\Omega_{t}^{1}\right)_{t}$ and $\left(\Omega_{t}^{2}\right)_{t}$ defined by $\left[18, \sqrt{19}\right.$ with $b_{\varepsilon} \equiv h_{+}^{\varepsilon}, a_{\varepsilon} \equiv h_{-}^{\varepsilon}, b=m_{+}$, $a=m_{-}$but with $\tau=0$, and $\Omega_{0}^{1}, \Omega_{0}^{2}$ defined by 22 .

We reformulate the key result of the very small time initialization to point out the main differences:

LEMMA 2.8 Under the assumptions of Theorem 2.4, for any $\beta>0$, there exists a constant $\tau>0$ such that if $t_{\varepsilon}=\tau \varepsilon$, then, for all sufficiently small $\varepsilon$,

$$
u_{\varepsilon}\left(x, t_{\varepsilon}\right) \geqslant\left(h_{+}^{\varepsilon}\left(x, t_{\varepsilon}\right)-\beta\right) \mathbb{1}_{\left\{d\left(x, t_{\varepsilon}\right) \geqslant \beta\right\}}+\left(h_{-}^{\varepsilon}\left(x, t_{\varepsilon}\right)-\beta\right) \mathbb{1}_{\left\{d\left(x, t_{\varepsilon}\right)<\beta\right\}} \quad \text { on } \bar{O} .
$$

As mentioned above, in the $\left(h_{+}^{\varepsilon}(x, t)-\beta\right)$ and $\left(h_{-}^{\varepsilon}(x, t)-\beta\right)$ terms, $\beta$ is now playing the role played above by $\beta \varepsilon$, and the dependence of $t_{\varepsilon}$ on $\varepsilon$ leads to simplification in the proof: for example, to prove Lemma 2.8 , we do not need any more to modify $f^{\varepsilon}$ in a complicated way and may work with the ode

$$
\dot{\chi}(\xi, s, x, t)+f^{\varepsilon}(\chi(\xi, s, x, t), x, t)+\beta \varphi\left(-C \beta^{-1} \bar{\delta}(x)\right)=0 .
$$

The point is that now the derivatives of $\chi$ with respect to $\xi, x$ and $t$ are bounded if $\beta$ is fixed for $s \leqslant a(\delta)$. On the other hand, the analogue of the function $\psi$ defined by (38) depends now on $x$ and $t$ and in order that the term $\psi(d(x, t), x, t)$ satisfies the Neumann boundary condition, we have to define it in the following way:

$$
\psi(z, x, t):=m_{0}(x, t)+\delta \varphi\left(C \delta^{-1} \bar{\delta}(x)\right)+\tilde{\psi}(z),
$$

where $\tilde{\psi}: \mathbb{R} \rightarrow \mathbb{R}$ is a smooth function such that $-C \leqslant \tilde{\psi} \leqslant \delta$ in $\mathbb{R}, \tilde{\psi}(z)=-C$ in $\{z<0\}$ and $\tilde{\psi}(z)=\delta$ on $\{z \geqslant \delta\}$. By taking $C>0$ large enough, the Neumann boundary condition is satisfied since $m_{0}(x, t)+\delta \varphi\left(C \delta^{-1} \bar{\delta}(x)\right)$ satisfies it, and we also have the key property $m_{0}(x, t)+$ $\delta \varphi\left(C \delta^{-1} \bar{\delta}(x)\right)-C \leqslant-\|g\|_{\infty}$ in $\bar{O} \times[0, \infty)$.

For the propagation, we argue in the same way but with a $v^{\varepsilon}$ of the form

$$
v^{\varepsilon}(x, t)=q^{\varepsilon}\left(\varepsilon^{-1}(d(x, t)-2 \beta)\right)-2 \beta \varphi\left(-C \beta^{-1} \bar{\delta}(x)\right),
$$

and $g_{ \pm}^{\varepsilon}$ are changed in an analogous way.

We leave the computations to the reader since they are simpler than those above.

Acknowledgements. This work was partially supported by the TMR program "Viscosity Solutions and Their Applications". It was partially done when the second author was visiting the Courant Institute of Mathematical Sciences; she would like to thank Professors Fang-Hua Lin and Robert V. Kohn for interesting discussions and all the Department for its kind hospitality. We are 
also grateful to Dejan Slepcev for pointing out an error in the formulation of Theorem 1.2. Last but not least, we want to thank very much the anonymous referee who, by an extremely detailed report, led us to find a much simpler formulation of the geometric definition of sub- and superflows, which resulted in far simpler proofs.

\section{REFERENCES}

1. Allen, S. M. \& CAHN, J. W. A macroscopic theory for antiphase boundary motion and its application to antiphase domain coarsening. Acta Metal. 27 (1979), 1085-1095.

2. Aronson, D. G. \& Weinberger, H. Multidimensional nonlinear diffusion arising in population genetics. Adv. Math. 30 (1978), 33-76. Zbl 0407.9201 MR 80a:35013

3. BARLES, G. Nonlinear Neumann boundary conditions for quasilinear degenerate elliptic equations and applications. J. Differential Equations 154 (1999), 191-224. Zbl 0924.35051 MR 2001c:35078

4. BARles, G. \& Georgelin, C. A simple proof for the convergence of an approximation scheme for computing mean curvature motions. SIAM J. Numer. Anal. 32 (1995), 484-500. Zbl 0831.65138 MR 96c:65140

5. Barles, G., Soner, H. M., \& Souganidis, P. E. Front propagation and phase field theory. SIAM J. Control. Optim. 31 (1993), 439-469. Zbl 0785.35049 MR 94c:35005

6. Barles, G. \& Souganidis, P. E. A new approach to front propagation problems: theory and applications. Arch. Ration. Mech. Anal. 141 (1998), 237-296. Zbl 0904.35034 MR 99c:35106

7. CHEN, X. Generation and propagation of interfaces in reaction-diffusion equations. J. Differential Equations 96 (1992), 116-141. Zbl0765.35024 MR 92m:35129

8. Chen, Y.-G., GigA, Y., \& Goto, S. Uniqueness and existence of viscosity solutions of generalized mean curvature flow equations. J. Differential Geom. 33 (1991), 749-786. Zbl 0696.35087 MR 93a:35093

9. Crandall, M. G., Ishit, H., \& Lions, P.-L. User's guide to viscosity solutions of second order partial differential equations. Bull. Amer. Math. Soc. 27 (1992), 1-67. Zbl 0755.35015 MR 92j:35050

10. Evans, L. C., Soner, H. M., \& Souganidis, P. E. Phase transitions and generalized motion by mean curvature. Comm. Pure Appl. Math. 45 (1992), 1097-1123. Zbl 0801.35045 MR 93g:35064

11. Evans, L. C. \& Spruck, J. Motion of level sets by mean curvature I. J. Differential Geom. 33 (1991), 635-681. Zbl 0726.53029 MR 92h:35097

12. FIFE, P. C. \& MCLEOD, B. The approach of solutions of nonlinear differential diffusion equations to traveling solutions. Arch. Ration. Mech. Anal. 65 (1977), 335-361. Zbl 0361.35035 MR 56 \#862

13. Fleming, W. H. \& Soner, H. M. Controlled Markov Processes and Viscosity Solutions. Appl. Math. 25, Springer, New York, 1993. Zbl 0773.60070 MR 94e:93004

14. GIGA, Y. Evolving curves with boundary conditions. Curvature flows and Related Topics (Levico Terme, 1994), A. Damlamian, J. Spruck and A. Visintin (eds.), Gakkōtosho (1995), 99-109. Zbl 0840.35019 MR 96j:3511

15. Giga, Y. \& SATo, M.-H. Neumann problem for singular degenerate parabolic equations. Differential Integral Equations 6 (1993), 1217-1230. Zbl 0806.35098 MR 94g:35129

16. IshiI, H. \& SATO, M.-H. Nonlinear oblique derivative problems for singular degenerate parabolic equations on a general domain. Preprint.

17. Katsoulakis, M. A., Kossioris, G., \& Reitich, F. Generalized motion by mean curvature with Neumann conditions and the Allen-Cahn model for phase transitions. J. Geom. Anal. 5 (1995), 255-279. Zbl 0827.35003 MR 96f:35183

18. Keller, J. B., Rubinstein, J., \& Sternberg, P. Fast reaction, slow diffusion and curve shortening. SIAM J. Appl. Math. 49 (1989), 116-133. Zbl 0701.35012 MR 89m:35117

19. Osher, S. \& Sethian, J. A. Fronts propagating with curvature-dependent speed: algorithms based on Hamilton-Jacobi formulations. J. Comput. Phys. 79 (1988), 12-49. Zbl 0659.65132 MR 89h:80012 
20. Soner, H. M. Motion of a set by the curvature of its boundary. J. Differential Equations 101 (1993), 313-372. Zbl 0769.35070 MR 94b:58038

21. Souganidis, P. E. Front propagation: theory and applications. Viscosity Solutions and Applications (Montecatini Terme, 1995), I. Capuzzo Dolcetta et al. (eds.), Lecture Notes in Math. 1660, Springer, Berlin (1997), 186-242. Zbl 0882.35016 MR 98g:35010

22. Souganidis, P. E. Interface dynamics in phase transitions. Proceeding ICM 94, Birkhäuser, Basel (1995), 1133-1144. Zbl 0845.35045 MR 97j:35074 